\title{
MODIFIED CYCLODEXTRIN MICROPARTICLES TO IMPROVE PMMA DRUG DELIVERY WITHOUT MECHANICAL LOSS
}

\author{
by \\ CHAO-YI LU \\ Submitted in partial fulfillment of the requirements for the degree of \\ Master of Science \\ Department of Biomedical Engineering \\ CASE WESTERN RESERVE UNIVERSITY
}

May, 2020 


\title{
CASE WESTERN RESERVE UNIVERSITY SCHOOL OF GRADUATE STUDIES
}

\author{
We hereby approve the thesis/dissertation of \\ Chao-yi Lu
}

candidate for the degree of Master of Science*.

\author{
Committee Chair \\ Horst von Recum, PhD
}

Committee Member

Agata Exner, PhD

Committee Member

Jon Pokorski, PhD

Date of Defense

March $13^{\text {th }}, 2020$

*We also certify that written approval has been obtained for any proprietary material contained therein. 


\section{TABLE OF CONTENTS}

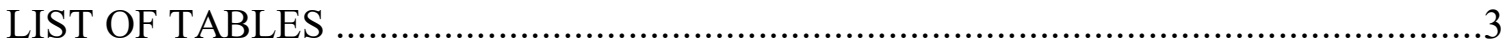

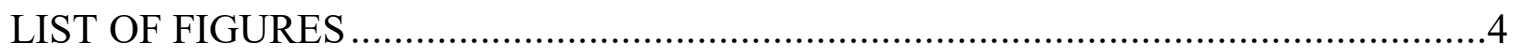

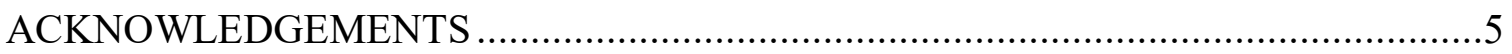

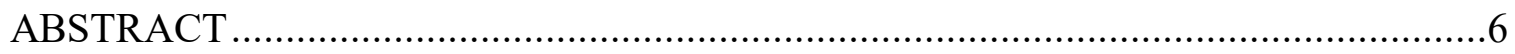

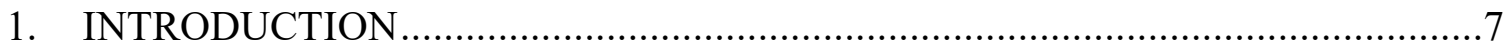

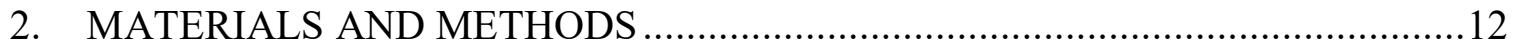

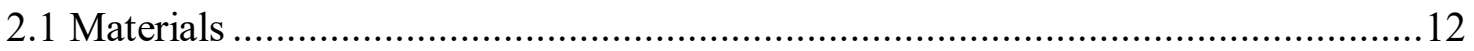

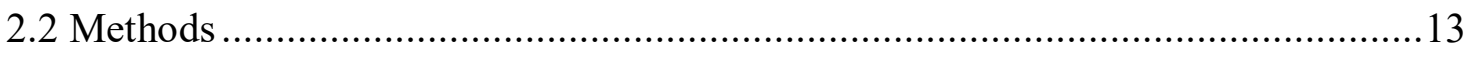

2.2.1 Preparation of insoluble polymerized cyclodextrin ( $\beta-C D)$ microparticles ......13

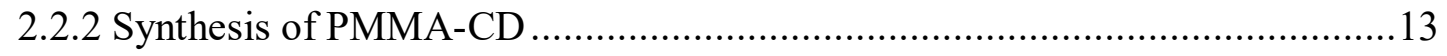

2.2.3 Synthesis of PMMA spherical beads and cylinders ………..........................15

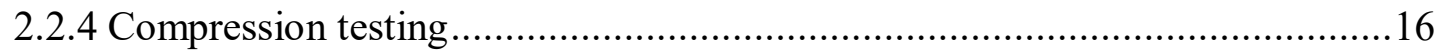

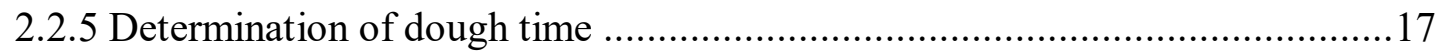

2.2.6 Measurement of temperature ....................................................................... 17

2.2.7 Determination of setting time and working time.........................................18

2.2.8 Filling RMP into microparticles .............................................................. 18

2.2.9 Quantification of drug in RMP-filled microparticles ....................................19

2.2.10 PMMA antibiotic-refilling in agarose phantom study ..................................19

2.2.11 Quantification of PMMA antibiotic refilling efficiency .................................20

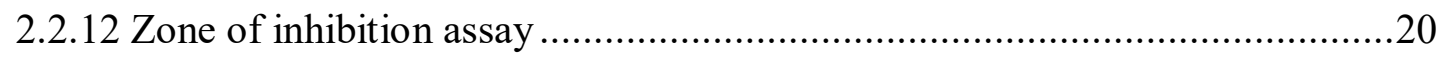

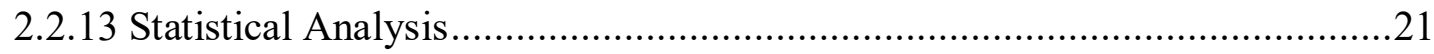

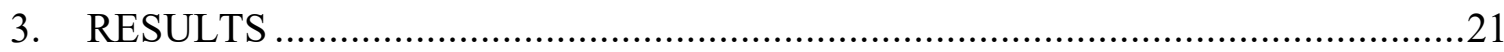

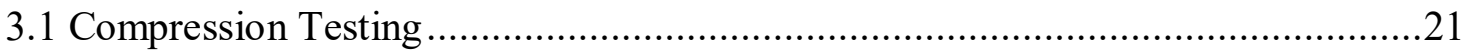

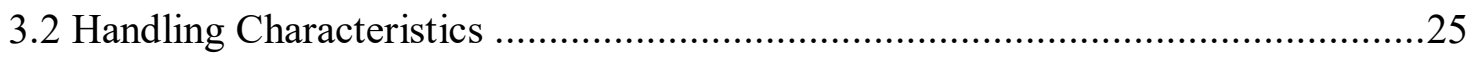

3.3 Quantification of RMP in RMP-filled microparticles........................................28

3.4 Refilling PMMA samples through agarose phantom model ..................................29

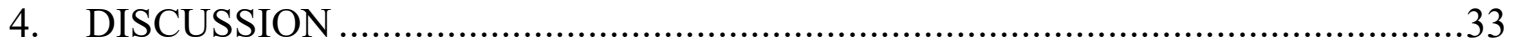

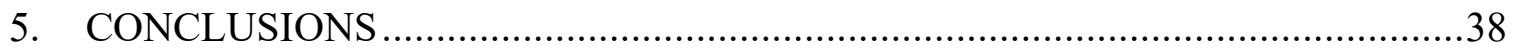

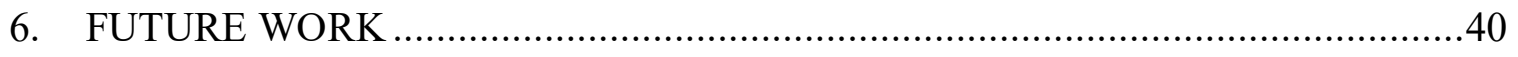

6.1 Investigating Other Properties of PMMA with CD Microparticles .........................40 
6.2 Improving Mechanical and Handling Properties of PMMA Containing CD

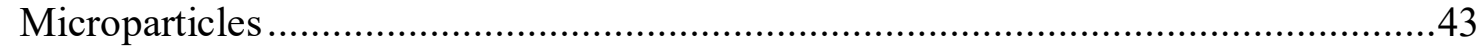

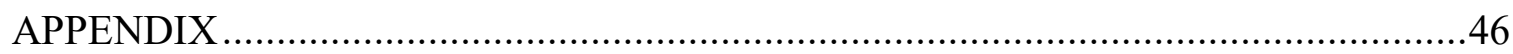

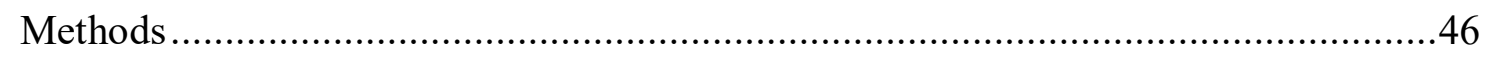

Scanning Electron Microscopy (SEM) Imaging ...............................................46

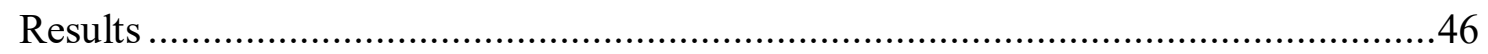

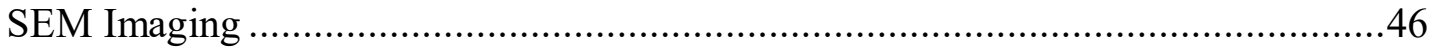

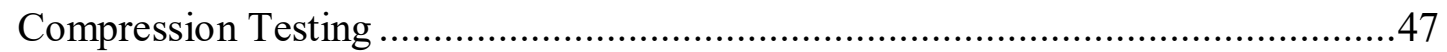

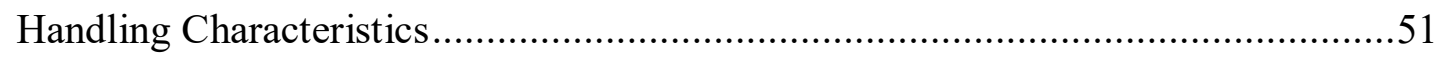

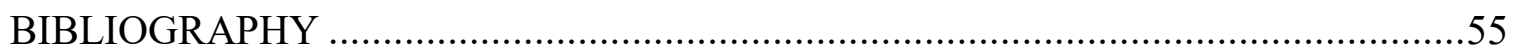




\section{LIST OF TABLES}

Table S1. Mechanical properties of PMMA samples without microparticles and PMMA samples with $10 \mathrm{wt} \% \mathrm{CD}$ microparticles (unmodified $\beta-\mathrm{CD}, 3.8 \mathrm{kDa}$ PMMA-CD, 12.2 kDa PMMA-CD, or 26.3 kDa PMMA-CD)

Table S2. Mechanical properties of plain PMMA without microparticles and PMMA samples with between 5 to $20 \mathrm{wt} \% \beta$-CD microparticles (unmodified $\beta$-CD or $26.3 \mathrm{kDa}$

PMMA-CD) 49 


\section{LIST OF FIGURES}

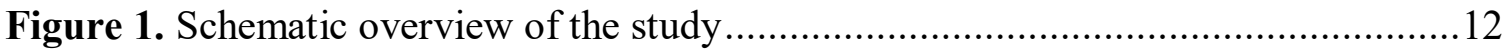

Figure 2. Synthesis of PMMA-CD. (a) Representative RAFT polymerization for PMMA-COOH (b) conjugation of PMMA-COOH onto $\beta$-CD microparticles.

Figure 3. Ultimate compressive strengths of PMMA samples containing $10 \mathrm{wt} \% \beta-\mathrm{CD}$ microparticles (unmodified $\beta$-CD without PMMA, CD with $3.8 \mathrm{kDa}$ PMMA, CD with 12.2 kDa PMMA, and CD with 26.3 kDa PMMA)

Figure 4. Relationship between ultimate compressive strengths and $\mathrm{wt} \%$ (ranging from 0 to $15 \mathrm{wt} \%$ ) of microparticles (unmodified $\beta$-CD microparticles and $26.3 \mathrm{kDa}$ PMMACD) added to PMMA

Figure 5. Handling characteristics of PMMA samples (plain PMMA without microparticles, PMMA with $10 \mathrm{wt} \%$ unmodified $\beta$-CD microparticles, and PMMA with $10 \mathrm{wt} \%$ 26.3kDa PMMA-CD microparticles). (a) Dough time, setting time, and working time of PMMA samples. (b) Maximum polymerization temperature and setting temperature of PMMA samples

Figure 6. Amount of rifampicin (RMP) that was leached from $20 \mathrm{mg}$ of RMP-filled CD microparticles (unmodified $\beta-\mathrm{CD}, 3.8 \mathrm{kDa}$ PMMA-CD, and $26.3 \mathrm{kDa}$ PMMA-CD) ......28

Figure 7. Images of refilled PMMA sample without $C D$ microparticles and PMMA samples with $10 \mathrm{wt} \% \beta-C D$ microparticles (i.e. unmodified $\beta-C D, 3.8 \mathrm{kDa}$ PMMA-CD,

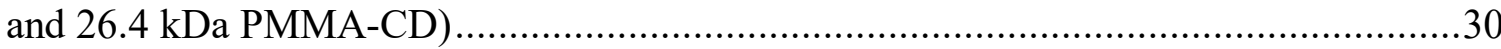

Figure 8. Amount of rifampicin (RMP) that was refilled into the PMMA samples (without microparticles and with between 5 to $15 \mathrm{wt} \%$ unmodified or modified (i.e. 3.8

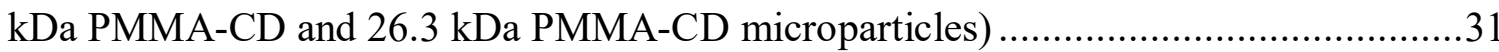

Figure 9. Zone of inhibition of refilled PMMA samples without microparticles, PMMA samples with $10 \mathrm{wt} \%$ unmodified $\beta$-CD microparticles, PMMA samples with $10 \mathrm{wt} \% 3.8$ kDa PMMA-CD, and PMMA with 10 wt\% 26.3 kDa PMMA-CD . .32

Figure 10. Image of push-out test system developed by our lab 42

Figure S1. Scanning Electron Microscopy (SEM) images of plain PMMA without microparticles, PMMA with $15 \mathrm{wt} \% \beta-\mathrm{CD}$, and PMMA with $15 \mathrm{wt} \% 26.3 \mathrm{kDa}$ PMMACD.

Figure S2. Ultimate compressive strengths of PMMA samples with between 0 to $20 \mathrm{wt} \%$ $\mathrm{CD}$ microparticles (unmodified $\beta$-CD microparticles or $26.3 \mathrm{kDa}$ PMMA-CD) at constant liquid monomer to solid ratio.

Figure S3. Representative temperature profiles of different PMMA formulations (plain PMMA without microparticles and PMMA with $10 \mathrm{wt} \%$ unmodified $\beta-C D$ microparticles). Temperature profiles of PMMA with $10 \mathrm{wt} \% 26.3 \mathrm{kDa}$ PMMA-CD microparticles are also shown 


\section{ACKNOWLEDGEMENTS}

This study would not have been possible with the support of many people. First of all, I would like to thank my advisor Dr. Horst von Recum for providing me the opportunity to work in his lab and for giving me guidance throughout this study.

I would also like to thank Dr. Jon Pokorski and Dr. Derek Church for their work and for synthesizing the modified cyclodextrin microparticles used in this study, Dr. Agata Exner for joining my committee, Greg Learn for assistance in the mechanical tests, Kathleen Young for assistance in the SEM imaging, Erika Cyphert for general feedback and suggestions for this study, and Zhe Ren for sputter-coating the PMMA samples.

Lastly, I want to thank all of my friends and family who supported and helped me during my time at Case Western Reserve University. 


\title{
Modified Cyclodextrin Microparticles to Improve PMMA Drug Delivery Without Mechanical Loss \\ By CHAO-YI LU
}

\begin{abstract}
Antibiotic-loaded poly(methyl methacrylate) (PMMA) cement is commonly used as a local delivery system to treat and prevent orthopedic infections associated with arthroplasties in load-bearing applications. However, these delivery systems are inefficient as release rate sharply declines to subinhibitory levels. Prior studies have shown that by adding in drug-filled cyclodextrin (CD) microparticles into PMMA cement, a more consistent release was observed. Additionally, antibiotic refilling through simulated implantation was achieved, but a loss in mechanical properties was noticed. In order to decrease the mechanical loss, modified CD microparticles (PMMA-CD) were synthesized. The compressive strengths, handling characteristics, and refilling ability of PMMA with PMMA-CD were evaluated. Up to a $10 \%$ increase in compressive strength was observed when CD was substituted with PMMA-CD. A longer working time and lower maximum temperature were observed with both the addition of CD and of PMMACD into PMMA, and refilling was also observed in PMMA with PMMA-CD.
\end{abstract}




\section{INTRODUCTION}

Over a million total joint arthroplasties are performed annually in the US, and the number is estimated to increase to around 4 million by 2030 (1). One major complication associated with arthroplasties is infection. Even though only around 1-3\% orthopedic surgeries develop postoperative implant-associated infections, conventional systemic antibiotic treatment may cost between $\$ 15,000$ and $\$ 30,000$ and may lead to further complications such as implant removal and extended hospitalization (2). Additionally, systemic antibiotics have limited efficacy since orthopedic infections are less likely to be well perfused or accessible systemically (3).

To treat and prevent these orthopedic infections more effectively, a local delivery system may be favored since it allows a higher local concentration of antibiotics near the point of infection while reducing the risks of systemic toxicity (3). One common practice for surgeons to create a local delivery system for treating orthopedic infections is to mix antibiotics into poly(methyl methacrylate) (PMMA) bone cement prior to application of cement (3-5). Antibiotic-loaded PMMA is used in a variety of applications, such as the temporary spacer during a two-stage revision surgery for total hip arthroplasty, the cement interfaced between the implant and native tissue in arthroplasty load-bearing applications, and the antibiotic beads for treating osteomyelitis (3). These uses of antibiotic-laden PMMA have been shown to be more effective in preventing and treating orthopedic infections than systemic antibiotic treatment. More specifically, the use of PMMA cement with antibiotic have successfully reduced infection rates from $7 \%$ to $1 \%$ for primary joint replacement surgeries (6) and from $12 \%$ to $3.7 \%$ for severe open fractures (3). 
Despite its success in decreasing orthopedic infection rates, antibiotic-laden PMMA has its limitations. One of which is the limited choices of antibiotics that can be used in this system. In order for an antibiotic to be compatible with the PMMA delivery system, the drug has to be: (1) soluble in water so that it can be released from the PMMA, (2) thermally stable in order to retain its activity after exposure of heat from PMMA polymerization, and (3) non-interfering to PMMA polymerization (7). Gentamicin exhibits all the qualities aforementioned and is one of the most common antibiotics added to PMMA (4). Other antibiotics that have been used in PMMA include tobramycin, vancomycin, erythromycin, and cefuroxime (8-11). However, this is a limited selection of drugs that can added into PMMA, and many of these drugs do not provide sufficient antimicrobial activity against some common bacterial strains associated with orthopedic infections. More specifically, gentamicin, tobramycin, and vancomycin have been observed to be less efficient against gram-negative bacterial strains which can account for up to $23 \%$ of periprosthetic joint infections (7).

Another limitation of antibiotic-laden PMMA is its biphasic drug release $(4,12)$.

Specifically, there is an initial peak release followed by a long period of low release at a subinhibitory concentration $(4,9)$. It has been observed that the local concentration of antibiotic drops below the therapeutic level within the first few days after surgery (13) which may be insufficient for treating or preventing orthopedic infections as only $45 \%$ of prosthetic infections occur in the first three months after surgery (14). Additionally, clinical studies have shown that around $80 \%$ of the drug remains within the PMMA after years (4). The prolonged drug release at a subinhibitory concentration also can lead to development of antibiotic resistance $(4,15)$. The short duration in antimicrobial activity 
achieved by antibiotic-laden PMMA suggests a need for a better drug delivery system for treating orthopedic infections that can provide prolonged antimicrobial activity. Many research has been done to improve the drug delivery of PMMA to allow a longer duration of antimicrobial effect, including loading drugs into liposomes (6), halloysite nanotubes (16), and mesoporous silica nanoparticles (14) prior to addition into PMMA. However, these systems also have limited antimicrobial activity, since the maximum amount of drug that can be released is limited by the initial amount inside the system prior to implantation as refilling of drug into the system post-implantation may be unfeasible. One viable option to achieve extension in antimicrobial activity through refilling of drug into the system is through the addition of crosslinked cyclodextrin microparticles into PMMA.

Cyclodextrins are cyclic oligosaccharides that are composed of between six to eight glucose units. They have torus structures with relatively hydrophilic exteriors and hydrophobic interiors which allow complexation with hydrophobic drugs through affinity-based interactions. Through these interactions, crosslinked cyclodextrins have shown to allow a more consistent and steadier release of antibiotics and enable refilling of newly administered drug into the system (17).

Similar trends were observed when crosslinked, insoluble $\beta$-CD microparticles were added into PMMA where drug is released at a steadier rate for a longer duration in comparison to plain PMMA. Experiments have also suggested the possibility of refilling antibiotics into PMMA containing $\beta-C D$ microparticles post-implantation, further extending the antimicrobial effect of the system. Furthermore, the $\beta$-CD microparticles permitted the incorporation of previously incompatible drug into the PMMA drug 
delivery system (18). The ability to refill makes the drug delivery system more effective against bacteria since it prolongs the antimicrobial effect and offers the possibility of incorporating new drugs into the system during the course of treatment.

One observation made with PMMA containing $\beta$-CD microparticles is the depreciation in its mechanical strength relative to clinically-used PMMA without microparticles. Specifically, the incorporation of $10 \mathrm{wt} \%$ empty $\beta$-CD microparticles into PMMA resulted in an approximately $25 \%$ decrease in ultimate compressive strength (18). The mechanical strength of the PMMA formulation can be of interest since one of the primary applications of PMMA cement is in load-bearing arthroplasties (19). The decrease in compressive strength upon addition of $\beta-\mathrm{CD}$ microparticles may limit the use of this PMMA formulation to non-loadbearing applications. It has been suggested that the PMMA samples with CD microparticles can be strengthened through interactions between the CD microparticles and the PMMA chains (20). One possibility is through chain entanglement. PMMA is synthesized through the mixing of prepolymerized PMMA beads or cement powder and liquid methyl methacrylate monomer. During polymerization, the newly formed PMMA chains entangle with the polymer chains in the PMMA cement powder $(4,21)$. Using a similar concept in this work, PMMA chains were crosslinked onto CD microparticles (PMMA-CD) to allow for interactions between the modified CD microparticles and the PMMA. It was hypothesized that the PMMA containing these modified CD microparticles would reduce the mechanical loss observed in PMMA containing unmodified $\beta-C D$ microparticles while maintaining the formulation's ability to refill post-implantation. 
In this study, modified CD microparticles with different PMMA chain weights (i.e. 3.8 $\mathrm{kDa}, 12.2 \mathrm{kDa}$, and $26.3 \mathrm{kDa}$ ) were synthesized. Mechanical tests were conducted to evaluate (1) the relationship between the compressive strengths and the PMMA chain weights on modified CD microparticles and (2) the effect modification of CD microparticles has on the relationship between the compressive strengths and the weight percentage of microparticles added. Another important aspect evaluated was the handling characteristics of the PMMA formulation. Complications of PMMA include aseptic loosening and thermal necrosis due to the exothermic reaction of polymerization $(11,22)$. Short handling time of PMMA is also one of the main factor for surgeries involving PMMA to fail as surgeons were unable to properly position implants before the setting of PMMA $(23,24)$. Therefore, the effect of adding CD microparticles, both unmodified and modified, into PMMA on the cement's handling time and temperature profile was investigated. Lastly, the ability to fill antibiotics into modified CD microparticles and the capacity of PMMA containing these CD microparticles to refill through antibiotic diffusion in agarose tissue-mimic were investigated. A zone of inhibition assay against Staphylococcus aureus, one of the most common bacteria strains associated with orthopedic infection, was performed to test the duration of the antimicrobial effect of the refilled PMMA samples (25).

The syntheses of CD microparticles and of PMMA samples along with evaluation methods for different PMMA formulations used in this study are summarized in Figure 1. 


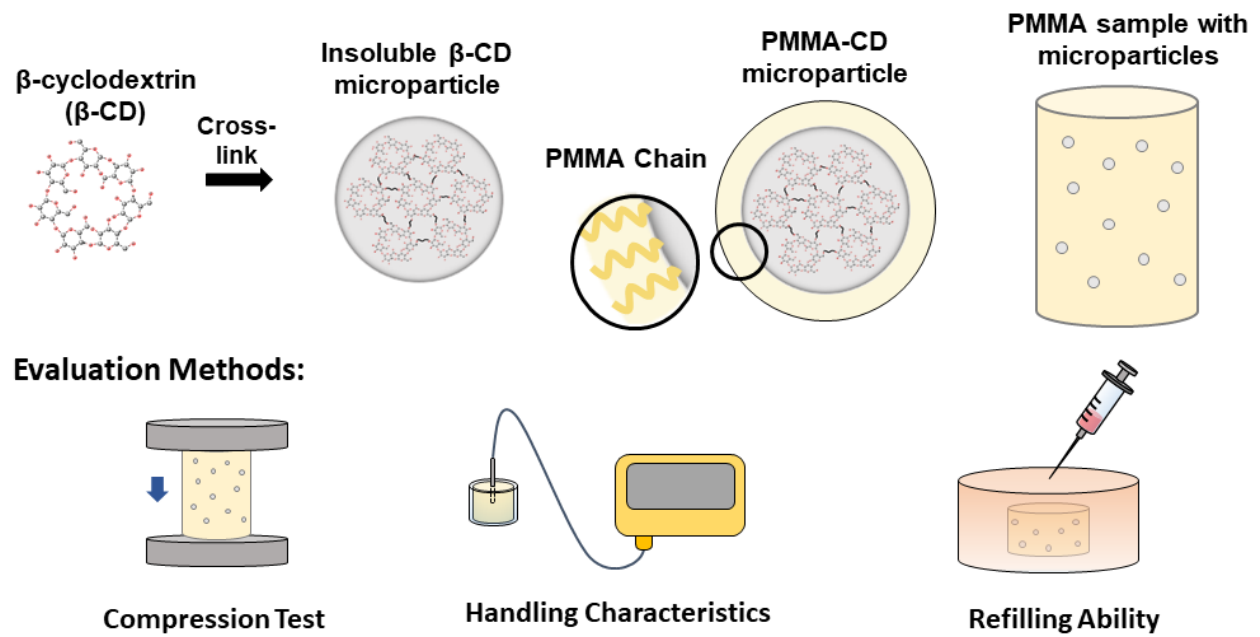

Figure 1. Schematic overview of the study. Unmodified CD microparticles and PMMA-CD microparticles were synthesized and mixed into PMMA. These PMMA samples were then evaluated for their mechanical properties, handling characteristics, and refilling abilities.

\section{MATERIALS AND METHODS}

\subsection{Materials}

Lightly epichlorohydrin cross-linked $\beta$-cyclodextrin $(\beta-C D)$ prepolymer was purchased from CycloLab (Budapest, Hungary). Ethylene glycol diglycidyl ether was purchased from Polysciences Inc. (Warrington, PA). Rifampicin (RMP) was purchased from Research Products International (Mt. Prospect, IL). Stryker ${ }^{\mathrm{TM}}$ Simplex $^{\circledR}$ HV (high viscosity) radiopaque bone cement produced by Stryker Orthopaedics (Mahwah, NJ) was purchased from eSutures. Poly(tetrafluoroethylene) (PTFE) stock for cylindrical specimen molds was purchased from McMaster-Carr (Aurora, OH). K-type thermocouple probes produced by PerfectPrime were purchased from Amazon (TL1004). A 4 channel K-type thermometer SD logger produced by Gain Express was purchased from Amazon (A0188598, Hong Kong). Green fluorescent protein (GFP)-labeled Staphylococcus 
aureus (S. aureus) stock culture was kindly provided by Dr. Edward Greenfield (Case Western Reserve University, $\mathrm{OH}$ ). All other reagents, solvent, and chemicals were purchased from Fisher Scientific in the highest grade available.

\subsection{Methods}

\subsubsection{Preparation of insoluble polymerized cyclodextrin $(\beta-C D)$ microparticles}

Cyclodextrin microparticles were synthesized based on previously established protocol (26,27). Briefly, $1.6 \mathrm{~mL}$ ethylene glycol diglycidyl ether crosslinker was added into $4 \mathrm{~mL}$ of dissolved lightly epichlorohydrin cross-linked $\beta$-cyclodextrin $(1 \mathrm{~g})$ in $0.2 \mathrm{M}$ potassium hydroxide. The polymer solution was then poured into a beaker containing $50 \mathrm{~mL}$ light mineral oil and $750 \mu \mathrm{L}$ of surfactant (Tween $85(24 \%)$ and Span $85(76 \%))$ heated at 60 ${ }^{\circ} \mathrm{C}$ and stirred at $850 \mathrm{rpm}$. The emulsion was kept at constant elevated temperature and stirring speed for the next 4 hours. The microparticles were then repeatedly washed and centrifuged for two times with solvents of increasing hydrophilicity (light mineral oil, hexane, acetone, MilliQ water). The microparticles were then frozen in MilliQ water before lyophilization.

\subsubsection{Synthesis of PMMA-CD}

Synthesis of PMMA-CD was performed by first synthesizing carboxyl-terminated PMMA (PMMA-COOH) then conjugating PMMA-COOH onto CD microparticles.

Figure 2 shows a schematic representation for this process. Through these processes, $\beta$ CD microparticles with different chain weights of PMMA (i.e. 3.8 kDa PMMA-CD, 12.2 kDa PMMA-CD, and 26.3 kDa PMMA-CD) were synthesized. 
(a)

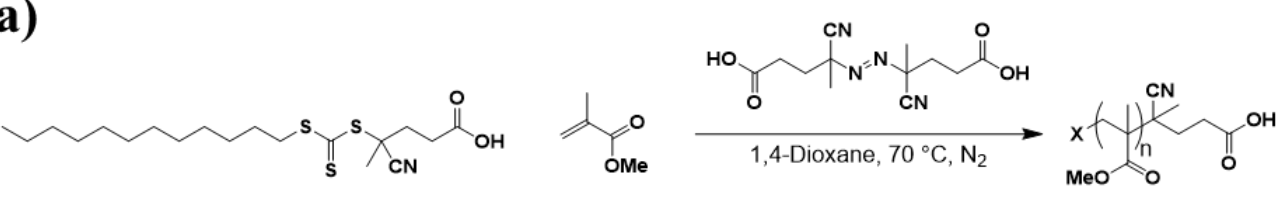

(b)
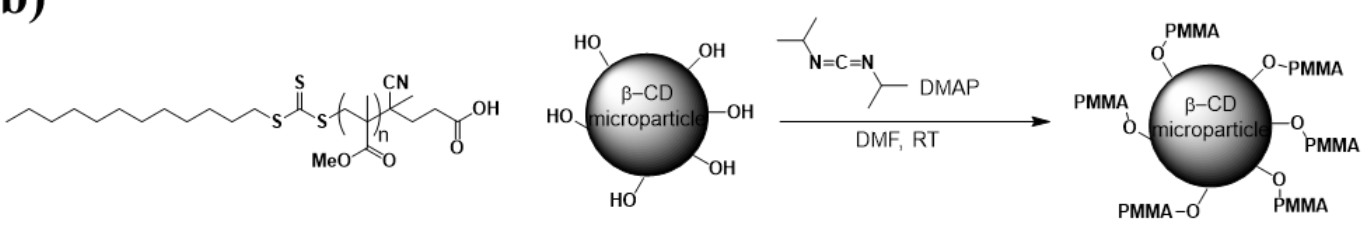

Figure 2. Synthesis of PMMA-CD. (a) Representative RAFT polymerization for PMMA-COOH (b) conjugation of PMMA-COOH onto $\beta-\mathrm{CD}$ microparticles.

RAFT (reversible addition-fragmentation chain transfer) polymerization was performed to synthesize PMMA-COOH. Figure 2a includes the schematic representation of this process. 4-Cyano-4-[(dodecylsulfanylthiocarbonyl)sulfanyl] pentanoic acid (100 mg, $0.25 \mathrm{mmol}, 1.0$ eq.), 4,4-Azobis (4-cyanovaleric acid) (7 mg, $0.025 \mathrm{mmol}, 0.1 \mathrm{eq}$.$) ,$ methyl methacrylate (7.9 mL, $74.32 \mathrm{mmol}, 300.0$ eq.), and $9.3 \mathrm{~mL}$ 1,4-dioxane were mixed in a flame-dried, $\mathrm{N}_{2}$-purged Schlenk flask using a stir bar. Freeze/pump/thaw were conducted to remove any residual oxygen from the reaction vessel. After the removal of oxygen, the vessel was then submerged into a preheated oil bath at $70^{\circ} \mathrm{C}$, and the solution was stirred for 15 hours. The flask was removed from heat and opened to air to terminate the polymerization. The reaction mixture was then diluted with $20 \mathrm{~mL}$ dioxane and precipitated dropwise into $400 \mathrm{~mL}$ methanol. The polymer was then collected as an offwhite powder (5.61 g, 78\% yield).

PMMA-COOH was then conjugated onto $\beta$-CD microparticles. $4.13 \mathrm{~g}$ of $\beta$-CD microparticles $\mathrm{N}, \mathrm{N}$-dimethylaminopyridine (183 $\mathrm{mg}, 1.50 \mathrm{mmol}, 35.0$ eq.) and $37 \mathrm{~mL}$ of N,N-dimethylformamide (DMF) were added into a flame-dried, $\mathrm{N}_{2}$-purged round bottom 
flask and mixed using a stir bar. In another flask, N,N'-diisopropylcarbodiimide $(0.23$ $\mathrm{mL}, 1.50 \mathrm{mmol}, 35.0$ eq.) was added to PMMA-COOH (1.12 g, $0.043 \mathrm{mmol}, 1.0 \mathrm{eq})$ in $11 \mathrm{~mL}$ dichloromethane and allowed to react for $10 \mathrm{~min}$. This polymer solution was then added to the $\beta$-CD microparticle suspension and stirred for $24 \mathrm{~h}$. The suspension was then washed by first centrifuging at $800 \mathrm{rpm}$ for $5 \mathrm{~min}$, removing the supernatant, and resuspending the pellet in $15 \mathrm{~mL}$ DMF. This procedure was conducted for a total of three times. The particles were then washed with $15 \mathrm{~mL}$ deionized $\mathrm{H}_{2} \mathrm{O}$ to remove residual DMF. After washing, the microparticles were stored in $15 \mathrm{~mL}$ deionized $\mathrm{H}_{2} \mathrm{O}$ and frozen at $-80{ }^{\circ} \mathrm{C}$. Lyophilization was then performed for $48 \mathrm{~h}$ to obtain PMMA-CD $(3.77 \mathrm{~g})$.

\subsubsection{Synthesis of PMMA spherical beads and cylinders}

PMMA spherical beads were prepared by mixing $1 \mathrm{~g}$ of Simplex ${ }^{\circledR}$ HV radiopaque bone cement powder with $500 \mu \mathrm{L}$ of methyl methacrylate liquid monomer, in accordance with manufacturer instructions. For PMMA samples with CD microparticles, the microparticles (i.e. unmodified $\beta$-CD, 3.8 kDa PMMA-CD, 12.2 kDa PMMA-CD, or 26.3 kDa PMMACD) were added to the bone cement powder prior to application of liquid monomer. The PMMA dough was then pressed into a thin sheet to form $6 \mathrm{~mm}$ diameter beads which were then left at room temperature to dry.

PMMA cylinders were synthesized based on previously established protocol (18). Briefly, $2 \mathrm{~g}$ of Simplex ${ }^{\circledR} \mathrm{HV}$ radiopaque bone cement powder was mixed with $1 \mathrm{~mL}$ of methyl methacrylate liquid monomer. CD microparticles (i.e. unmodified $\beta-\mathrm{CD}, 3.8 \mathrm{kDa}$ PMMACD, 12.2 kDa PMMA-CD, 26.3 kDa PMMA-CD) were added to the bone cement powder prior to application of liquid monomer for samples with microparticles. The PMMA dough 
was then pressed into a custom-machined two-part PTFE mold to form cylindrical samples $6 \mathrm{~mm}$ in diameter and $12 \mathrm{~mm}$ in height in accordance to the ASTM F451-16 (28).

\subsubsection{Compression testing}

Compression tests were performed to determine the ultimate compressive strengths of PMMA samples and how they vary with: 1) the PMMA chain weights on modified CD microparticles and 2) the amount of CD microparticles present in the PMMA samples. Observations were also made to study the effect modification of CD microparticles had on the correlation between the amount of microparticles added and the compressive strengths of PMMA samples.

PMMA samples with $10 \mathrm{wt} \%$ (equivalent to $200 \mathrm{mg}$ of microparticles for $2 \mathrm{~g}$ of prepolymerized PMMA cement powder) CD microparticles (i.e. unmodified $\beta$-CD, 3.8 kDa PMMA-CD, and 26.3 kDa PMMA-CD) were fabricated to determine the relationship between the PMMA chain weights on modified CD microparticles and the compressive strengths of PMMA samples. For evaluating the relationship between the amount of CD microparticles added into PMMA samples and the compressive strengths of samples, PMMA samples with $5 \mathrm{wt} \%, 10 \mathrm{wt} \%$, and $15 \mathrm{wt} \% \mathrm{CD}$ microparticles (i.e. unmodified $\beta$ $\mathrm{CD}$ and $26.3 \mathrm{kDa}$ PMMA-CD) were prepared (equivalent to $100 \mathrm{mg}, 200 \mathrm{mg}$, and $300 \mathrm{mg}$ of microparticles, respectively, for $2 \mathrm{~g}$ of prepolymerized PMMA cement powder).

Sample preparation and compression tests were performed based on previously established protocol (20). Briefly, prior to the tests, the ends of cylindrical PMMA samples were sanded smooth using a wet 240 grit silicon carbide sandpaper on a drill press. Dimensions (i.e. diameter and length) of each PMMA samples were measured using a digital caliper to 
the nearest $0.01 \mathrm{~mm}$. Compression tests were performed using a mechanical testing frame (Material Testing System MTS 810, MTS Systems Corporation) with a 2000 lbf (8896 N) load cell approximately 48 hours after the initial synthesis of cylindrical PMMA samples. Samples were loaded at a rate of $20 \mathrm{~mm} / \mathrm{min}$ with a sampling rate of $200 \mathrm{~Hz}$. Loaddisplacement curves were obtained and used to evaluate the ultimate compressive strength, elastic modulus, work to peak load, strain to peak load, and absorbed energy per unit volume. These properties were determined using the $2 \%$ offset method when a local maximum was not present.

\subsubsection{Determination of dough time}

To evaluate the handling characteristics of PMMA with different microparticles, dough time determination and temperature profiling were completed using plain PMMA without microparticles and PMMA samples with $10 \mathrm{wt} \%$ microparticles (unmodified $\beta$-CD or 26.3 kDa PMMA-CD). Dough time was determined based on ASTM F451-16 (28). Prior to tests, all materials and equipment were stored at room temperature for at least $2 \mathrm{~h}$. A timer was started upon the addition of the liquid monomer into the PMMA cement powder at a 1:2 ratio $(\mathrm{mL} / \mathrm{g})$. After $60 \mathrm{~s}$ of mixing, probing was done with a gloved finger for every 15 s until the mixture separated off the glove cleanly. The dough was then further tested for temperature profiling.

\subsubsection{Measurement of temperature}

Within 1 min after the dough time, the dough was hand packed into one well of a Falcon ${ }^{\circledR}$ Multiwell $^{\mathrm{TM}} 24$ well tissue culture plate. A K-type thermocouple temperature probe 
(TL1004, PerfectPrime) was inserted $10 \mathrm{~mm}$ or halfway through the center of the well. Temperature was recorded at $0.5 \mathrm{~Hz}$ using a 4 channel $\mathrm{K}$-type thermocouple logger (A0188598, Gain Express, Hong Kong). Recording was stopped when cooling was observed.

\subsubsection{Determination of setting time and working time}

Determination of setting time was conducted in accordance to ASTM F461-16 (28). It was found based on the time when the temperature of the cement reached the setting temperature $\left(\mathrm{T}_{\text {set }}\right)$ which was calculated through the following equation:

$$
T_{\text {set }}=\frac{1}{2}\left(T_{\text {max }}+T_{\text {ambient }}\right)
$$

where $T_{\max }$ is the maximum temperature observed during the polymerization and $T_{\text {ambient }}$ is the ambient temperature. The working time was determined by subtracting the dough time from the setting time (29).

\subsubsection{Filling RMP into microparticles}

To determine the maximum amount of drug that can complex with a given amount of microparticles, RMP was used to fill different types of microparticles (unmodified $\beta$-CD, $3.8 \mathrm{kDa}$ PMMA-CD, or $26.3 \mathrm{kDa}$ PMMA-CD). $20 \mathrm{mg}$ microparticles ( $\beta-\mathrm{CD}, 3.8 \mathrm{kDa}$ PMMA-CD, or $26.3 \mathrm{kDa}$ PMMA-CD) were placed in $1.5 \mathrm{~mL}$ Eppendorf tubes. $1 \mathrm{~mL}$ of 25 $\mathrm{mg} / \mathrm{mL}$ RMP in DMF was added into each tube. These were vortexed and placed on the rotisserie for 3 days at room temperature. The microparticles were then centrifuged for 5 min and washed with MilliQ water for 5 times. These were then frozen and lyophilized for 3 days. Quantification of the amount of RMP was then performed. 


\subsubsection{Quantification of drug in RMP-filled microparticles}

To quantify the amount of drug that was loaded into the different microparticles, the lyophilized drug-loaded microparticles were then soaked in $1 \mathrm{~mL}$ DMSO and agitated at $37^{\circ} \mathrm{C}$ for 3 days to leach out RMP. Aliquots were taken and read at $473 \mathrm{~nm}$ using Biotek ${ }^{\mathrm{TM}}$ 96-well plate reader (H1: Winooski, VT). A standard curve was used to determine the concentration of RMP.

\subsubsection{PMMA antibiotic-refilling in agarose phantom study}

A tissue-mimicking agarose phantom model was used to evaluate the ability for PMMA samples with different types of CD to refill through simulated implantation. The agarose phantom model prepared using a previously established protocol (26,30). Briefly, agarose in PBS $(0.075 \mathrm{wt} / \mathrm{vol} \%)$ was brought to boil. While hot, $5 \mathrm{~mL}$ of the solution was added into individual wells of a Costar ${ }^{\mathrm{TM}}$ 6-well cell culture plate (Corning Life Sciences, Corning NY). Spherical PMMA beads were placed on top of the solidified agarose. Another $5 \mathrm{~mL}$ of hot agarose in PBS solution was added on top of the solidified agarose. Once solidified, a $6 \mathrm{~mm}$ hole was punched through the center of the upper solidified agarose. Area scans were then taken using a Biotek ${ }^{\mathrm{TM}}$ 96-well plate reader (19x19 point area scan; $473 \mathrm{~nm}$ ) to determine the background absorbance signal of the agarose with the PMMA bead. These were done using plain PMMA samples without CD and PMMA samples with $5 \mathrm{wt} \%, 10 \mathrm{wt} \%$, and $15 \mathrm{wt} \%$ of $\mathrm{CD}$ microparticles (unmodified $\beta$-CD, 3.8 kDa PMMA-CD, and 26.3 kDa PMMA-CD). 


\subsubsection{Quantification of PMMA antibiotic refilling efficiency}

To quantify the amount of RMP that was refilled into the PMMA bead samples through the agarose phantom model, PMMA spherical beads that were refilled with RMP were fully submerged in $2 \mathrm{~mL}$ DMSO. These were agitated at $37{ }^{\circ} \mathrm{C}$ for three days before aliquots were taken to determine the concentration of RMP using absorbance spectroscopy at $473 \mathrm{~nm}$.

\subsubsection{Zone of inhibition assay}

A zone of inhibition assay was conducted with the PMMA beads (PMMA without microparticles, PMMA with $10 \mathrm{wt} \%$ unmodified $\beta$-CD, PMMA with $10 \mathrm{wt} \% 3.8 \mathrm{kDa}$ PMMA-CD, and PMMA with $10 \mathrm{wt} \% 26.3 \mathrm{kDa}$ PMMA-CD) to determine the duration of their antimicrobial activity after refilling. This assay is performed based on a previously established protocol (26). Briefly, $70 \mu \mathrm{L}$ Staphylococcus aureus was spread over Trypticase soy agar in a $100 \mathrm{~mm}$ diameter Petri dish. A refilled PMMA bead was then

placed on the center of the bacteria-covered agar. This agar plate was then stored overnight in an incubator at $37^{\circ} \mathrm{C}$. Measurements for the zone of inhibition of the refilled beads were taken the following day using calipers and were taken from the edge of the sample to the edge of clearance. Four readings were taken 90 degrees from each other for each PMMA bead and were averaged for each bead. Each condition was completed in triplicates. After measurement of the zone of clearance, the PMMA bead was then transferred on to another freshly seeded agar plate. The assay was performed for a total of 14 days. 


\subsubsection{Statistical Analysis}

All experiments were done with at least 3 samples for each condition $(n \geq 3)$, and the average and standard deviation of each condition are displayed. Error bars are depicted as the standard deviation. All statistical tests were performed through Microsoft Excel 2016. For compression tests, one-tailed Student's t-tests with unequal variances were performed to determine the significance of difference between two conditions. Two-tailed Student's t-test with unequal variances were conducted to analyze the handling characteristics (dough time, setting time, working time, maximum temperature, and setting temperature), the quantification of RMP in microparticles and in refilled PMMA samples, and the zone of inhibition assay with refilled PMMA samples. P-values less than 0.05 were considered statistically significant.

\section{RESULTS}

\subsection{Compression Testing}

Ultimate compressive strengths of PMMA samples were tested to investigate the relationship between the PMMA chain weights on the microparticles and the compressive strength of samples. Results are shown in Figure 3. Compression tests were also performed to investigate the relationship between the weight percentage of microparticles added and the compressive strength of PMMA samples. The results are shown in Figure 4.

More specifically, Figure 3 shows the results from compressing PMMA samples that contained $10 \mathrm{wt} \% \mathrm{CD}$ microparticles (unmodified $\beta-\mathrm{CD}, 3.8 \mathrm{kDa}$ PMMA-CD, $12.2 \mathrm{kDa}$ PMMA-CD, and 26.3 kDa PMMA-CD). All samples had strengths significantly lower 
than plain PMMA which had a compressive strength of $72.4 \pm 2.2 \mathrm{MPa}$ (see Appendix) $(\mathrm{p}<0.05)$. PMMA samples with unmodified $\beta$-CD microparticles had the lowest ultimate compressive strength of 54.6 $\pm 2.6 \mathrm{MPa}$, whereas PMMA samples with $26.3 \mathrm{kDa}$ PMMA-CD had the highest compressive strength of $62.1 \pm 1.5 \mathrm{MPa}$. PMMA samples with $3.8 \mathrm{kDa}$ and $12.2 \mathrm{kDa}$ PMMA-CD had intermediate compressive strengths of $58.9 \pm$ 2.9 MPa and $60.9 \pm 2.8 \mathrm{MPa}$, respectively. A general positive correlation was observed where samples containing microparticles with longer PMMA chains had higher ultimate compressive strengths. The ultimate compressive strengths of PMMA samples with either $26.3 \mathrm{kDa}$ or $12.2 \mathrm{kDa}$ PMMA-CD microparticles were found to be significantly different from PMMA samples with unmodified $\beta$-CD microparticles $(\mathrm{p}<0.05)$. No significance was found between samples with $3.8 \mathrm{kDa}$ PMMA-CD microparticles and those with unmodified CD microparticles ( $\mathrm{p}>0.05)$. 


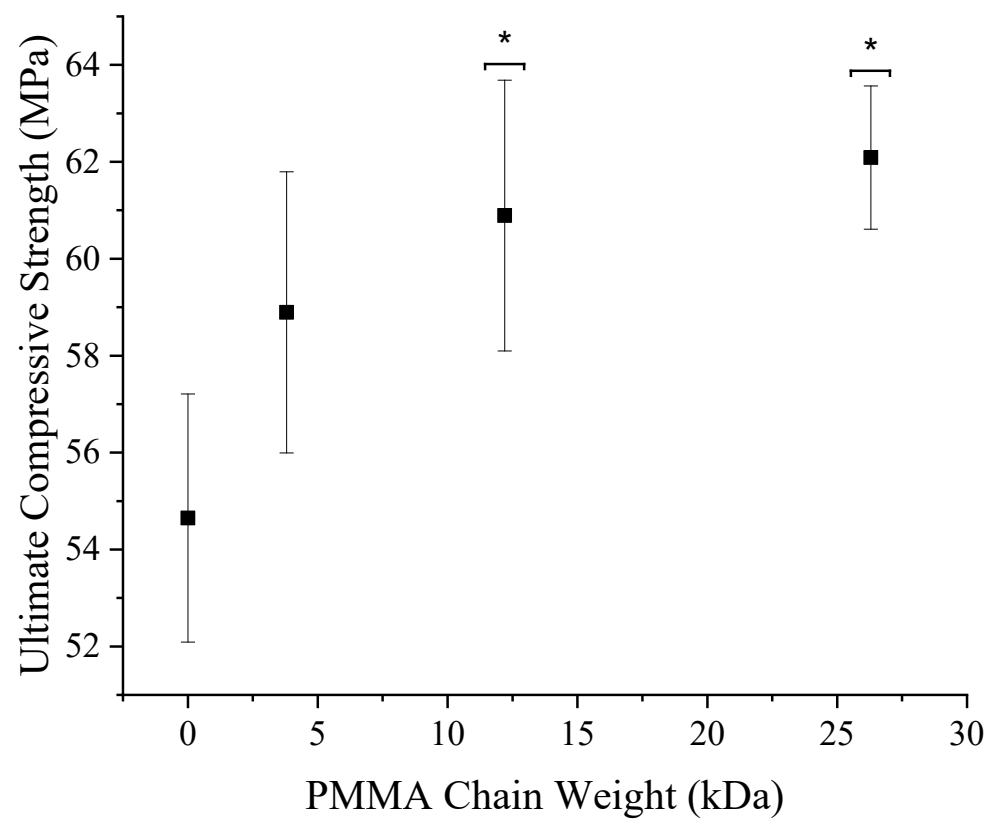

Figure 3. Ultimate compressive strengths of PMMA samples containing $10 \mathrm{wt} \% \beta-\mathrm{CD}$ microparticles with different PMMA chain lengths (unmodified $\beta-C D$ without PMMA, CD with 3.8 kDa PMMA, CD with 12.2 kDa PMMA, and CD with $26.3 \mathrm{kDa}$ PMMA) (n $\geq 3$ ). Statistically significance relative to PMMA samples with unmodified $\beta$-CD microparticles $(* \mathrm{p}<0.05)$. 


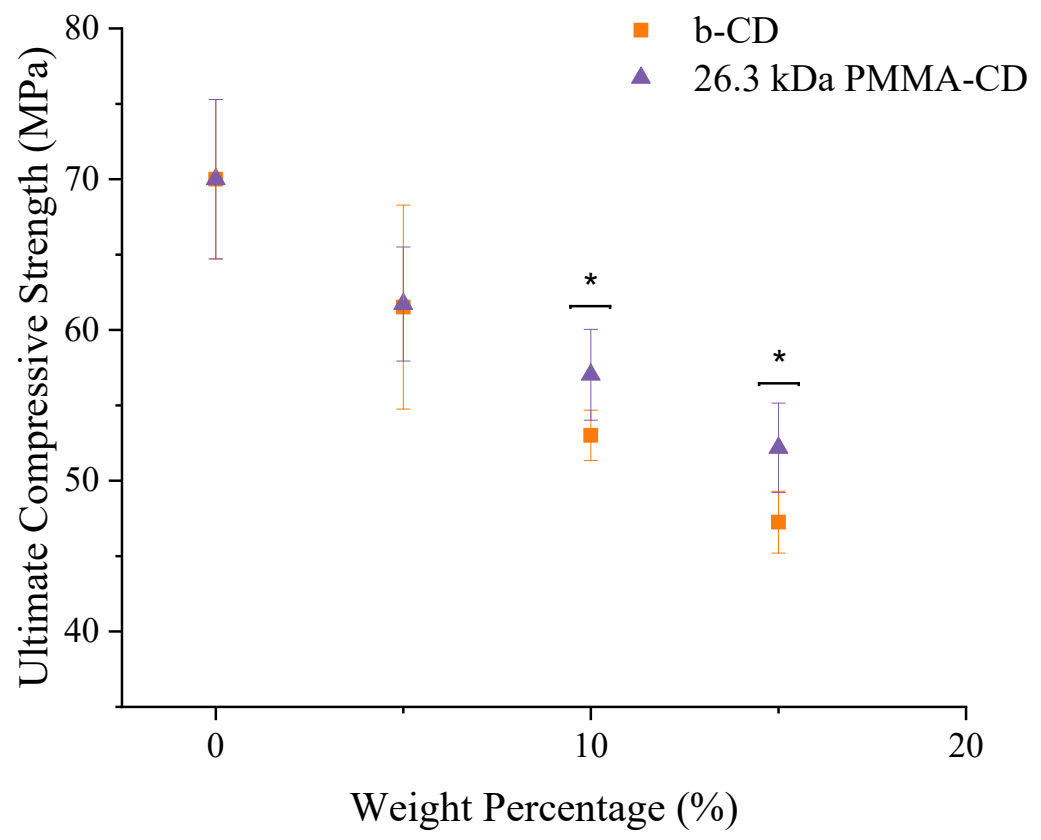

Figure 4. Relationship between ultimate compressive strengths and wt $\%$ (ranging from 0 to 15 $w t \%)$ of microparticles added for both PMMA samples with unmodified $\beta$-CD microparticles and PMMA samples with 26.3 kDa PMMA-CD ( $\mathrm{n} \geq 3$ ). Statistically significant difference between PMMA samples with same wt \% of CD microparticles (unmodified $\beta$-CD versus $26.3 \mathrm{kDa}$ PMMA-CD) $(* \mathrm{p}<0.05)$.

Ultimate compressive strengths of PMMA samples with addition of different weight percentages of CD microparticles were also tested. The results are shown in Figure 4. A general negative trend was observed where the higher amount of microparticles resulted in a lower ultimate compressive strength regardless of the type of CD microparticles that were added (i.e. unmodified $\beta-\mathrm{CD}$ microparticles and $26.3 \mathrm{kDa}$ PMMA-CD). However, PMMA samples with 26.3 kDa PMMA-CD tended to have higher compressive strengths than samples with the same amount of unmodified $\beta$-CD microparticles. This relationship became more apparent with increasing amount of 
microparticles in the formulation. At $5 \mathrm{wt} \% \mathrm{CD}$ microparticles added, both samples (PMMA with unmodified $\beta$-CD microparticles and PMMA with 26.3 kDa PMMA-CD) had ultimate compressive strengths of around $62 \mathrm{MPa}$. No significant difference was found between the two formulations ( $\mathrm{p}>0.05)$. With the addition of $15 \mathrm{wt} \%$ unmodified $\beta$-CD microparticles, the ultimate compressive strength of PMMA decreased to $47.3 \pm$ 2.1 MPa while the PMMA samples with $15 \mathrm{wt} \% 26.3 \mathrm{kDa}$ PMMA-CD had a compressive strength of 52.2 $\pm 3.0 \mathrm{MPa}$. With higher content of CD microparticles present in PMMA samples (i.e. $10 \mathrm{wt} \%$ and $15 \mathrm{wt} \%$ ), the compressive strengths of PMMA samples were significantly different between PMMA samples with unmodified $\beta$-CD and samples with $26.3 \mathrm{kDa}$ PMMA-CD $(\mathrm{p}<0.05)$.

\subsection{Handling Characteristics}

Handling characteristics of different PMMA formulations (PMMA without microparticles, PMMA with $10 \mathrm{wt} \%$ unmodified $\beta-\mathrm{CD}$ microparticles, and PMMA with $10 \mathrm{wt} \% 26.3 \mathrm{kDa}$ PMMA-CD microparticles) were evaluated. The results are shown in Figure 5. Figure 5a depicts the dough time, the setting time, and the working time of the samples, and Figure $5 \mathrm{~b}$ shows the maximum polymerization temperature and the setting temperature of the samples.

With the addition of $10 \mathrm{wt} \%$ unmodified $\beta$-CD microparticles into PMMA, the average dough time of PMMA significantly decreased from $172 \mathrm{~s}$ to $122 \mathrm{~s}(\mathrm{p}<0.05)$ in comparison to plain PMMA without CD microparticles, while the average setting time increased from $670 \mathrm{~s}$ to $770 \mathrm{~s}(\mathrm{p}>0.05 ; \mathrm{p}=0.051)$. PMMA samples with $10 \mathrm{wt} \% 26.3$ 
kDa PMMA-CD microparticles had an intermediate average dough time of $130 \mathrm{~s}$ and an average setting time of $694 \mathrm{~s}$.

The addition of $10 \mathrm{wt} \%$ unmodified $\beta$-CD microparticles into PMMA also significantly decreased the average maximum polymerization temperature and average setting temperature from $77.4{ }^{\circ} \mathrm{C}$ to $71.0{ }^{\circ} \mathrm{C}$ and from $48.2^{\circ} \mathrm{C}$ to $45.7^{\circ} \mathrm{C}$, respectively $(\mathrm{p}<0.05)$ relative to plain PMMA without any microparticles. PMMA samples with $10 \mathrm{wt} \% 26.3$ kDa PMMA-CD had an intermediate average maximum polymerization temperature of $71.6^{\circ} \mathrm{C}$ and an average setting temperature of $45.7^{\circ} \mathrm{C}$. 


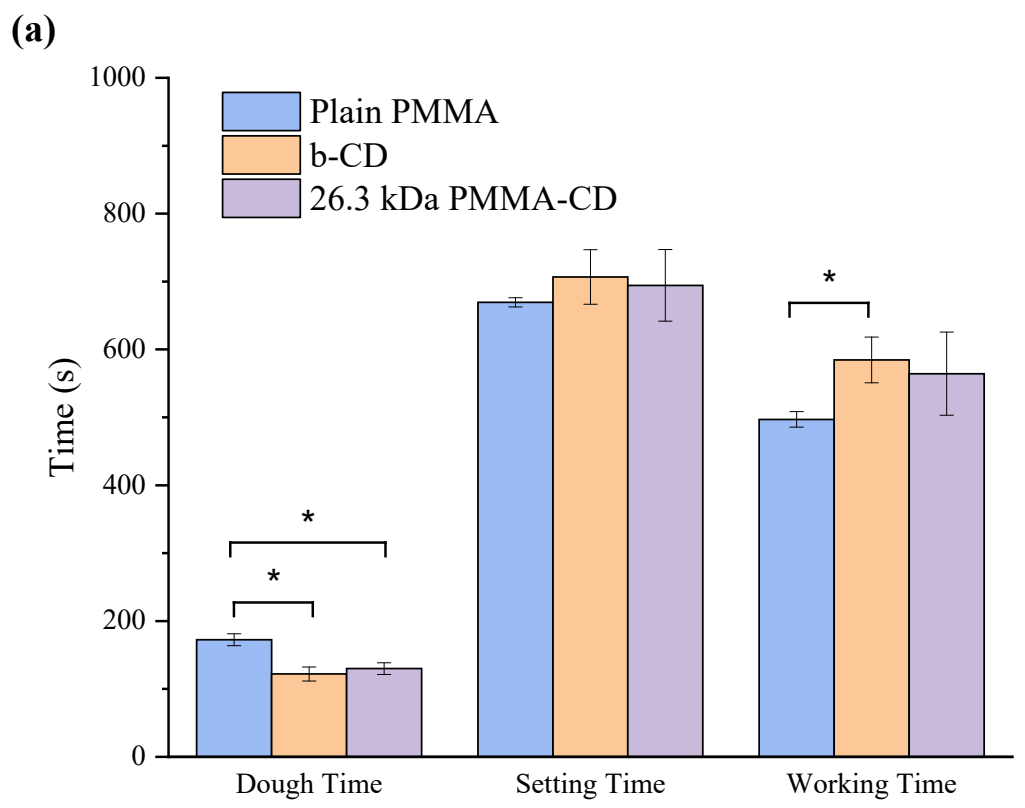

(b)

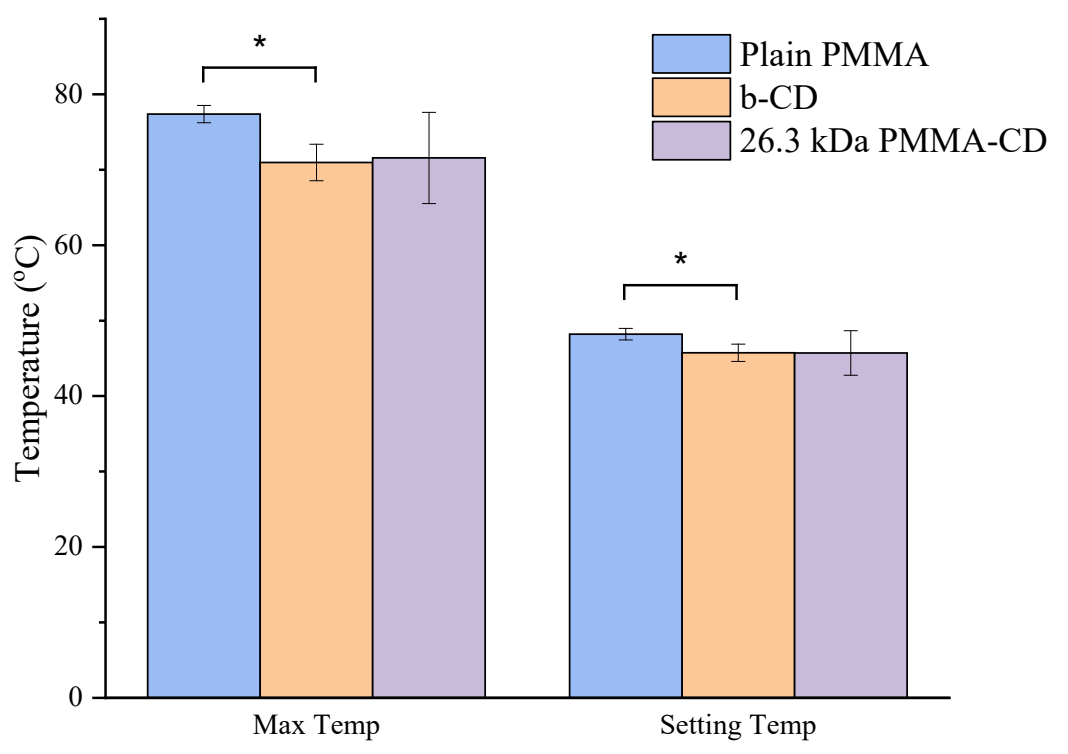

Figure 5. Handling characteristics of PMMA samples (plain PMMA without microparticles, PMMA with $10 \mathrm{wt} \%$ unmodified $\beta$-CD microparticles, and PMMA with $10 \mathrm{wt} \% 26.3 \mathrm{kDa}$ PMMA-CD microparticles). (a) Dough time, setting time, and working time of PMMA samples. 
(b) Maximum polymerization temperature and setting temperature of PMMA samples. ( $n=4$ for plain PMMA, $\mathrm{n}=7$ for $\beta-\mathrm{CD}, \mathrm{n}=3$ for PMMA-CD, $\left.{ }^{*} \mathrm{p}<0.05\right)$.

\subsection{Quantification of RMP in RMP-filled microparticles}

To determine the maximum amount of RMP that could complex with modified and unmodified CD microparticles, $20 \mathrm{mg}$ of microparticles (unmodified $\beta$-CD microparticles, 3.8 kDa PMMA-CD microparticles, and 26.3 kDa PMMA-CD microparticles) were filled with RMP and subsequently soaked in DMSO to leach out the drug. The amount of RMP that was leached out for different types of microparticles are represented in Figure 6.

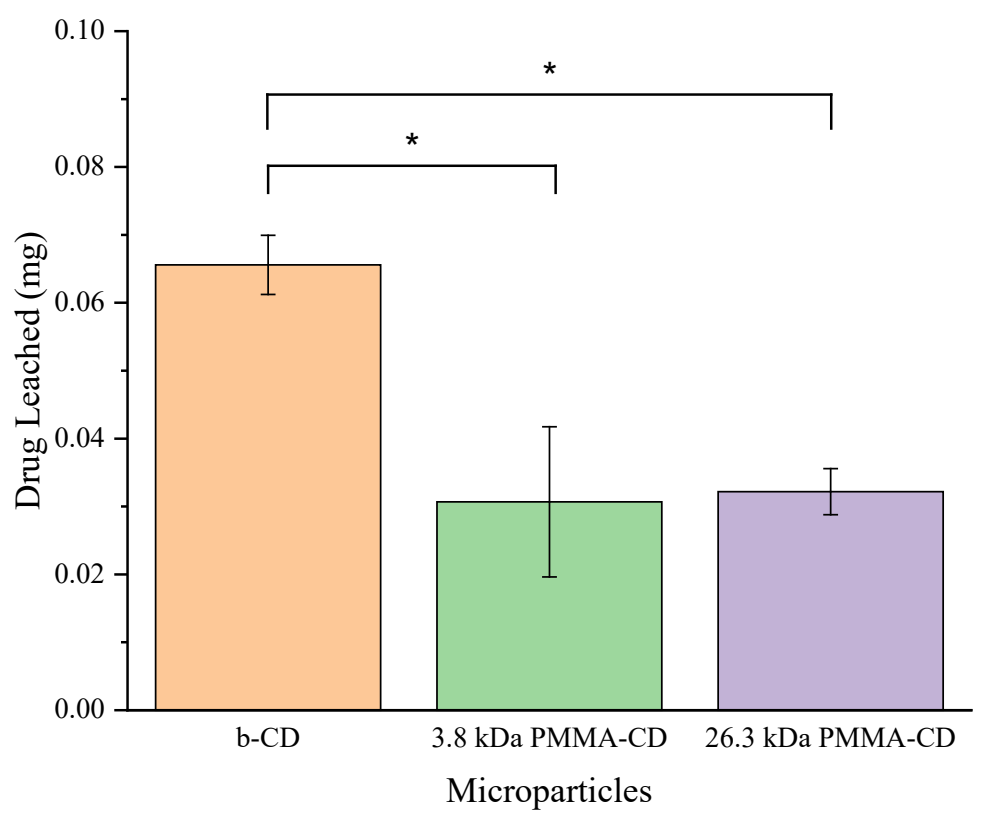

Figure 6. Amount of rifampicin (RMP) that was leached from $20 \mathrm{mg}$ of cyclodextrin (CD) microparticles (unmodified $\beta-\mathrm{CD}, 3.8 \mathrm{kDa}$ PMMA-CD, and 26.3 kDa PMMA-CD) that were filled with RMP. $(\mathrm{n} \geq 3, * \mathrm{p}<0.05)$. 
Out of all three types of CD microparticles, unmodified $\beta$-CD microparticles had the most amount of RMP leached out, averaging $66 \pm 4 \mu \mathrm{g}$. The modified CD microparticles, 3.8 kDa PMMA-CD and 26.3 kDa PMMA-CD, had similar amounts of RMP leached out, averaging $31 \pm 11 \mu \mathrm{g}$ and $32 \pm 3 \mu \mathrm{g}$, respectively. Significance was found between amount of RMP leached out from unmodified $\beta$-CD microparticles and that of RMP leached out from modified $\beta$-CD microparticles (i.e. $3.8 \mathrm{kDa}$ PMMA-CD and $26.3 \mathrm{kDa}$ PMMA-CD) $(\mathrm{p}<0.05)$. No significant difference was found for amount of drug leached out from the two modified $\beta$-CD microparticles (i.e. $3.8 \mathrm{kDa}$ PMMA-CD and $26.3 \mathrm{kDa}$ PMMA-CD) $(\mathrm{p}>0.05)$.

\subsection{Refilling PMMA samples through agarose phantom model}

To investigate the potential for PMMA samples containing CD microparticles to refill with antibiotic post implantation, PMMA samples with between 0 to $15 \mathrm{wt} \%$ of different types of CD microparticles (i.e. unmodified $\beta-\mathrm{CD}, 3.8 \mathrm{kDa}$ PMMA-CD, and $26.3 \mathrm{kDa}$ PMMA-CD) were refilled with RMP through the agarose phantom model. Figure 7 shows images of refilled PMMA samples containing no microparticles and PMMA samples with $10 \mathrm{wt} \%$ of $\mathrm{CD}$ microparticles (unmodified $\beta-\mathrm{CD}, 3.8 \mathrm{kDa}$ PMMA-CD, and 26.3 kDa PMMA-CD). Amount of RMP can be determined qualitatively through the "redness" of the PMMA samples, due to the red color of RMP. The "redness" of the refilled PMMA bead with $10 \mathrm{wt} \%$ unmodified $\beta$-CD microparticles suggested more RMP was refilled into this PMMA sample compared to others. Refilled PMMA beads with 10 wt\% modified CD microparticles (i.e. 3.8 kDa PMMA-CD and 26.3 kDa PMMA-CD) were of similar "redness" that was intermediate between refilled plain PMMA samples 
without microparticles and refilled PMMA samples with $10 \mathrm{wt} \%$ unmodified $\beta-C D$ microparticles.

The amount of RMP refilled into PMMA samples through the agarose phantom model was also determined quantitatively. Figure 8 depicts the normalized amount of RMP that was leached out from PMMA samples with between 0 to $20 \mathrm{wt} \%$ of $\beta$-CD microparticles (i.e. unmodified $\beta-\mathrm{CD}, 3.8 \mathrm{kDa}$ PMMA-CD, and $26.3 \mathrm{kDa}$ PMMA-CD).

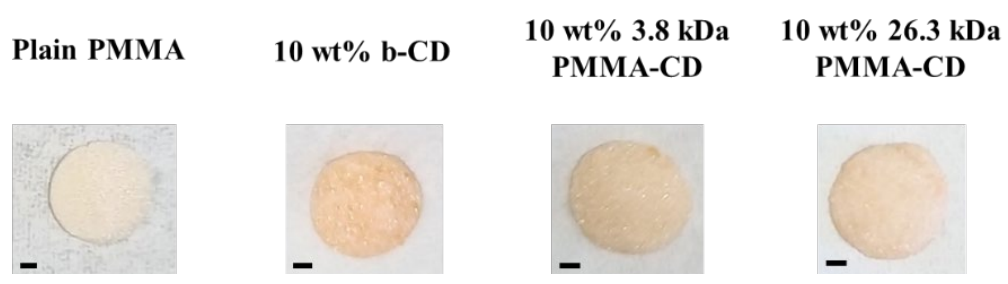

Figure 7. Images of refilled PMMA sample without $C D$ microparticles and PMMA samples with $10 \mathrm{wt} \% \beta$-CD microparticles (i.e. unmodified $\beta$-CD, $3.8 \mathrm{kDa}$ PMMA-CD, and $26.4 \mathrm{kDa}$ PMMACD). Samples were refilled with rifampicin (RMP) through the agarose phantom model. Scale bars $=1 \mathrm{~mm}$. 


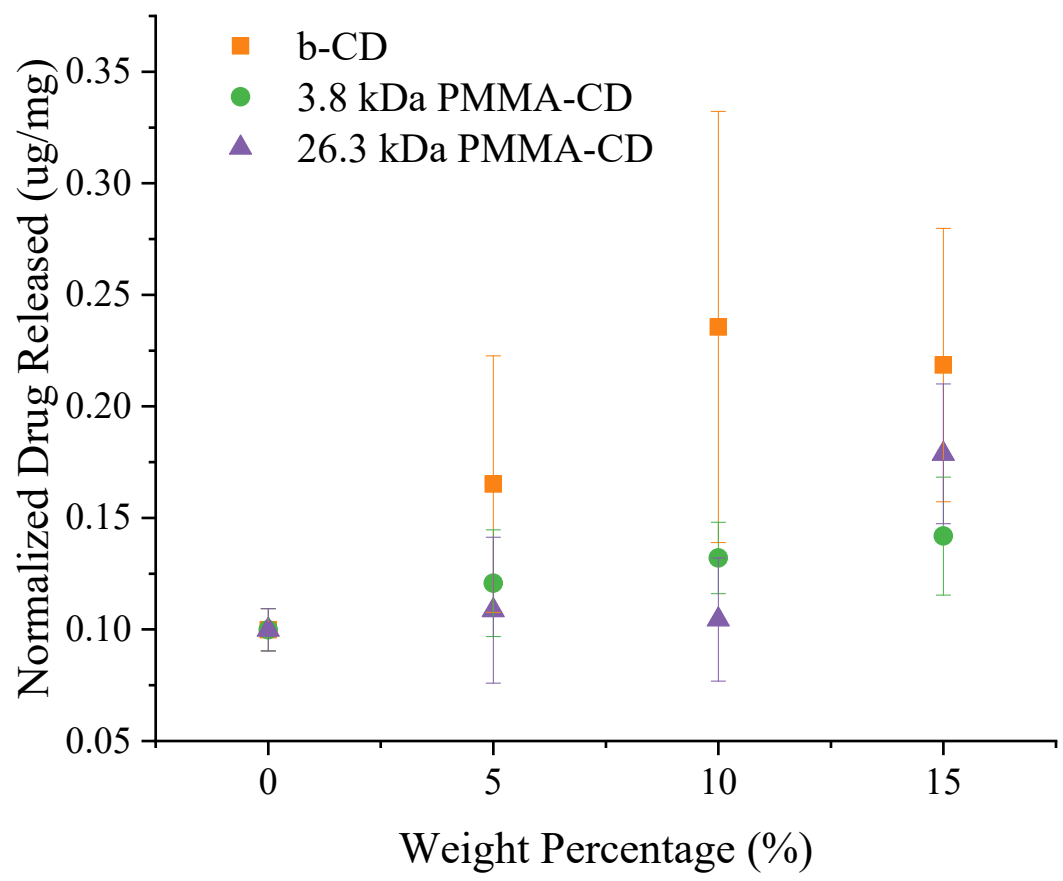

Figure 8. Amount of rifampicin (RMP) that was refilled into the PMMA samples (with between 0 to $15 \mathrm{wt} \%$ unmodified or modified (i.e. $3.8 \mathrm{kDa}$ PMMA-CD and $26.3 \mathrm{kDa}$ PMMA-CD microparticles) through the agarose phantom model $(n \geqslant 3)$.

Through quantification of RMP that was leached from refilled PMMA beads, general positive trends were observed where higher weight percentages of CD microparticles led to increase in amount of RMP that was leached out. PMMA samples with unmodified $\beta$ CD microparticles leached out the most amount of RMP compared to PMMA samples with the corresponding weight percentages of modified $\beta$-CD microparticles (i.e. $3.8 \mathrm{kDa}$ PMMA-CD and 26.3 kDa PMMA-CD). Significant difference was only observed at 10 wt $\%$ CD microparticles when comparing the amount of RMP leached out from PMMA samples with unmodified $\beta$-CD microparticles and amount of RMP leached out from 
PMMA samples with modified $\beta$-CD microparticles (i.e. $3.8 \mathrm{kDa}$ PMMA-CD and 26.3 kDa PMMA-CD) $(\mathrm{p}<0.05)$.

The duration of antimicrobial activity of refilled PMMA samples was evaluated through the zone of inhibition assay against $S$. aureus. Results are shown in Figure 9.

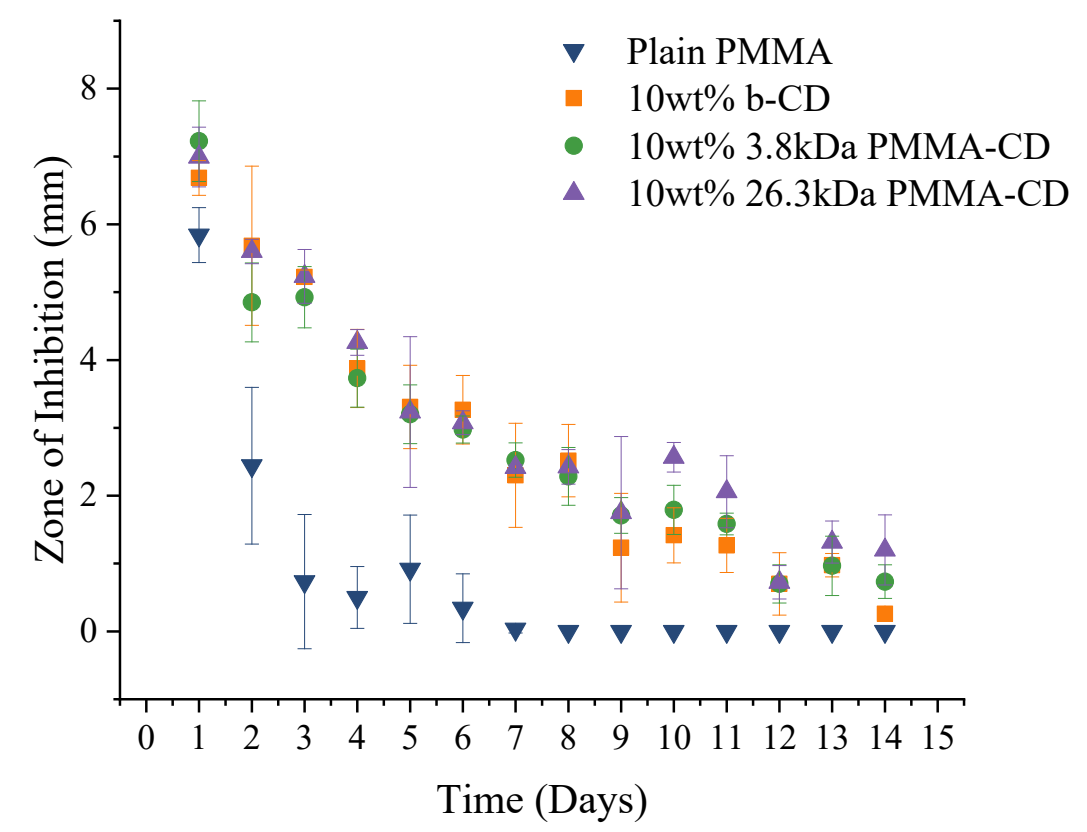

Figure 9. Zone of inhibition of refilled PMMA samples without microparticles ( $\mathbf{\nabla})$, PMMA samples with $10 \mathrm{wt} \%$ unmodified $\beta$-CD microparticles (-), PMMA samples with $10 \mathrm{wt} \% 3.8 \mathrm{kDa}$

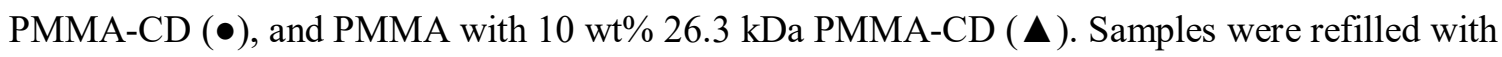
RMP for 48 hours through the phantom agarose refilling model, and their antimicrobial effects against S. aureus for 14 days were evaluated $(\mathrm{n}=3)$.

In general, refilled PMMA samples with CD microparticles (both unmodified and modified) had larger zones of clearance and longer antimicrobial durations in comparison to refilled plain PMMA samples without any microparticles. Specifically, no visible zone of clearance could be observed after seven days for refilled plain PMMA samples 
whereas the antimicrobial activities of refilled PMMA samples with CD microparticles lasted throughout the study (i.e. 14 days). Statistical analysis showed that there was mostly no significant difference in zone of clearance between PMMA samples with 10 wt $\%$ unmodified $\beta-C D$ microparticles and those with $10 \mathrm{wt} \%$ modified $\mathrm{CD}$ microparticles (i.e. 3.8 kDa PMMA-CD and 26.3 kDa PMMA-CD) $(\mathrm{p}>0.05$; except on Day 10 for $10 \mathrm{wt} \% 26.3 \mathrm{kDa}$ PMMA-CD). Additionally, the zones of inhibition for refilled plain PMMA samples were found to be significantly different from the zones of inhibition for refilled PMMA samples with $10 \mathrm{wt} \% \mathrm{CD}$ microparticles (i.e. unmodified $\beta$ CD microparticles, 3.8 kDa PMMA-CD, 26.3 kDa PMMA-CD) ( $p<0.05$; except on Days 9, 12-14).

\section{DISCUSSION}

In this study, PMMA samples with modified CD microparticles (i.e. PMMA-CD) were synthesized to decrease the loss in mechanical properties observed in PMMA cement samples containing unmodified $\beta-\mathrm{CD}$ microparticles. The handling characteristics and refilling ability of these PMMA formulations were also evaluated.

Mechanical tests were performed to investigate the relationship between PMMA chain weights on modified CD microparticles and ultimate compressive strengths of PMMA samples with CD microparticles. With the addition of $10 \mathrm{wt} \%$ unmodified $\beta-\mathrm{CD}$, PMMA lost around $25 \%$ of its mechanical strength. The number decreased to around $15 \%$ with the substitution of unmodified $\beta-\mathrm{CD}$ to $26.3 \mathrm{kDa}$ PMMA-CD. A general positive trend between the PMMA chain weights on CD microparticles and the compressive strengths of PMMA samples was observed where increasing PMMA chain weight resulted in a 
higher compressive strength. This may be due to the interaction (i.e. chain entanglement) between the PMMA chains synthesized onto the CD microparticles and the newly polymerized PMMA chains. Another possible explanation could be the presence of less CD microparticles as addition of microparticles was done in weight percentages instead of in number of $\mathrm{CD}$ microparticles. Another interesting observation made was the diminishing rate of increase in compressive strengths as longer PMMA chains were added onto the $\mathrm{CD}$ microparticles. This may be due to the difficulty for liquid monomer to penetrate through larger molecules (i.e. modified CD microparticles with higher molecular weights of PMMA) to allow more chain entanglement between the microparticles and PMMA.

Mechanical tests were also performed to evaluate the effect modification on CD microparticles had on the relationship between weight percentage of microparticles added and ultimate compressive strength of PMMA samples. A negative correlation was found between the ultimate compressive strength and the weight percentage of microparticles that were incorporated into the system regardless of the type of $\mathrm{CD}$ microparticles (i.e. unmodified $\beta-\mathrm{CD}$ or $26.3 \mathrm{kDa}$ PMMA-CD). Interestingly, with an increase in amount of CD microparticles added, the difference between the ultimate compressive strengths of PMMA samples with unmodified CD and those with $26.3 \mathrm{kDa}$ PMMA-CD became more apparent. Specifically, the compressive strengths of PMMA samples with $5 \mathrm{wt} \% \mathrm{CD}$ microparticles only increased by $0.3 \%$ with the replacement of unmodified $\beta-C D$ microparticles with 26.3 kDa PMMA-CD whereas the compressive strengths increased by $7.6 \%$ and $10.4 \%$ at $10 \mathrm{wt} \%$ and $15 \mathrm{wt} \% \mathrm{CD}$ microparticles added, correspondingly. The insignificant difference $(\mathrm{p}>0.05)$ in compressive strengths observed at $5 \mathrm{wt} \% \mathrm{CD}$ 
microparticles may be attributed to the presence of too little microparticles for a noticeable difference in ultimate compressive strengths. When higher amounts of CD microparticles were included (i.e. 10 and $15 \mathrm{wt} \% \mathrm{CD}$ microparticles), statistically significant differences $(\mathrm{p}<0.05)$ were observed between the PMMA samples with unmodified $\beta-\mathrm{CD}$ and those with $26.3 \mathrm{kDa}$ PMMA-CD. This may be attributed to presence of chain entanglement between the modified CD microparticles with PMMA to strengthen the sample. Another possible explanation is the presence of less CD microparticles since the addition of microparticles was done by weight instead of by number of microparticles.

In addition to examining the mechanical properties of the PMMA samples, temperature profiling was also done to investigate the handling characteristics of these samples. Overall, an increase in working time and a decrease in maximum polymerization temperature were observed in both PMMA samples with unmodified $\beta$-CD microparticles and those with modified CD microparticles (i.e. 26.3 kDa PMMA-CD). Others have observed that an increase in prepolymerized PMMA cement powder to liquid monomer ratio will lead to a decrease in setting time and some minor difference in dough time (31-33). In this study, we observed an increase in working time due to the general decrease in the dough time and increase in the setting time of PMMA samples that either contained the unmodified $\beta$-CD microparticles or contained the modified CD microparticles (i.e. 26.3 kDa PMMA-CD) which is different from the expected outcome for a PMMA cement with a higher solid to liquid monomer ratio. This deviation may suggest the presence of interaction between the $\mathrm{CD}$ microparticles and the liquid monomer that affects the polymerization of PMMA. The difference in setting time may 
also be due to the presence of larger molecules (i.e. CD microparticles) as studies have pointed out that it is more difficult for the liquid monomer to penetrate through larger molecules, thereby increasing the setting time when larger molecules are present in the system (32). Aside from the increase in the working time, it has been observed that the addition of CD microparticles (both modified and unmodified) also decreased the maximum polymerization temperature. This may also be another consequence of difficulty in wetting the system with having a higher solid to liquid monomer ratio in PMMA samples with CD microparticles $(32,33)$. Interestingly, no significant differences in their handling characteristics (i.e. working time and maximum polymerization temperature) were observed between PMMA samples that had unmodified $\beta$-CD microparticles and those that had 26.3 kDa PMMA-CD which may be due to the similarities in their liquid to solid ratios. In general, PMMA samples with modified CD microparticles had intermediate handling properties in comparison to plain PMMA samples and PMMA samples with unmodified $\beta$-CD microparticles which may be due to the lower content of $\mathrm{CD}$ microparticles interacting with the polymerization of the liquid monomer. The better handling characteristics (i.e. longer working time and lower maximum polymerization temperature) of PMMA samples with CD microparticles, both modified and unmodified, may be able to reduce the risk of aseptic loosening due to time restraint for proper placement of implant and thermal necrosis due to the exothermic polymerization of PMMA cement $(22,23)$. Further enhancement in handling properties could be done through adjustment of the liquid to solid ratio, the ambient temperature, or the temperature at which the liquid monomer was stored at (31). 
The refilling ability of PMMA samples with modified CD microparticles (i.e. $3.8 \mathrm{kDa}$ PMMA-CD and 26.3 kDa PMMA-CD) was also investigated through agarose phantom model. Through this study, it has been observed that modified CD microparticles were able to retain their ability to interact with antibiotics. A general positive trend was observed where increasing amount of RMP was able to be refilled into the PMMA sample with more CD microparticles, both unmodified and modified. One observation from this study is that PMMA samples with unmodified $\beta$-CD microparticles generally had more RMP refilled than PMMA samples with the same wt $\%$ of modified CD microparticles. This was expected as quantification of RMP leached out from microparticles showed similar results where around two times the amount of RMP could be filled into unmodified $\beta$-CD microparticles in comparison to modified CD microparticles (i.e. $3.8 \mathrm{kDa}$ PMMA-CD and 26.3 kDa PMMA-CD). This may be due to the presence of less CD microparticles when they are modified since leaching studies were done with the same weights of microparticles instead of similar number of microparticles. Another possible explanation is that the PMMA chains attached to the modified CD microparticles may have interfered with the interaction between the antibiotic and the CD microparticles.

In addition to quantification of RMP refilled into PMMA samples with modified CD microparticles, zone of inhibition studies were also conducted to investigate the duration of their antimicrobial effects after refilling. Interestingly, comparable antimicrobial activities were observed between PMMA samples with unmodified $\beta$-CD microparticles and those with modified CD microparticles (i.e. 3.8 kDa PMMA-CD and $26.3 \mathrm{kDa}$ PMMA-CD) despite their differences in amount of RMP that could be refilled into the 
samples. This could be explained by the zone of clearance measurement being more related to the amount of antibiotic released from the surface of the bead in contact with the bacteria and less related to the total amount of RMP that was refilled into the system. The contact surface of the bead onto the agar plate may be too small to detect the difference in antimicrobial activities between PMMA samples with unmodified $\beta$-CD microparticles and those with modified CD microparticles. Through the refilling models and zone of inhibition studies, PMMA samples with modified CD microparticles were shown to be able to be refilled with antibiotics while retaining the antimicrobial effect of the drugs. These are beneficial properties of the system to treat and prevent prolonged periprosthetic joint infections as antimicrobial effects can be further extended through addition of more or different antibiotics into the system. CD microparticles have been shown to be able to interact with a variety of drugs which suggests that PMMA samples with modified CD microparticles could also be used in a patient-specific therapy where different and more suitable drugs are used and refilled as the disease progresses (20).

\section{CONCLUSIONS}

In this study, modified CD microparticles (PMMA-CD) were added into PMMA to reduce the mechanical loss observed in PMMA formulations with unmodified $\beta$-CD microparticles while retaining the refilling capacity of the system. The mechanical properties of PMMA samples with varying weight percentages of unmodified $\beta$-CD microparticles and of PMMA-CD were evaluated. The compression tests revealed significant differences in the ultimate compressive strengths between PMMA samples 
with unmodified $\beta-C D$ microparticles and those with PMMA-CD at higher weight percentages (i.e. 10 and $15 \mathrm{wt} \%$ ) of $\mathrm{CD}$ microparticles added. Even though the compressive strengths of PMMA with PMMA-CD microparticles were still lower than that of plain PMMA without microparticles, an improvement in the mechanical properties with the replacement of unmodified $\beta-C D$ microparticles to modified (i.e. PMMA-CD) microparticles was observed. This result matched the goal of this study which is to reduce the mechanical loss observed with addition of unmodified $\beta$-CD microparticles into PMMA through the substitution of unmodified microparticles to PMMA-CD. The change in mechanical properties between the two formulations suggests the possibility of chain entanglement between the PMMA-CD microparticles and the PMMA. With the increase in mechanical properties, PMMA that contain PMMA-CD microparticles may be more amenable for use in load-bearing applications in comparison to PMMA that contain unmodified $\beta$-CD microparticles. Handling characteristics of PMMA samples with unmodified $\beta-\mathrm{CD}$ and samples with 26.3 $\mathrm{kDa}$ PMMA-CD were also evaluated. The addition of both modified and unmodified CD microparticles led to better handling properties (i.e. longer working time and lower maximum polymerization temperature) which may lower risks of aseptic loosening of the implant and thermal necrosis due to the exothermic polymerization of PMMA. Lastly, refilling abilities of PMMA samples with PMMA-CD were compared with samples with unmodified $\beta$-CD microparticles. Although more antibiotics were able to be refilled into PMMA samples with unmodified $\beta$-CD microparticles, the duration of antimicrobial activity of refilled PMMA samples with PMMA-CD were found to be comparable to those of refilled samples with unmodified microparticles. The refilling study also revealed the possibility for PMMA 
with PMMA-CD to have prolonged antimicrobial activity and patient-specific therapy that can help treat and prevent periprosthetic joint infections more effectively.

\section{FUTURE WORK}

This study has focused on evaluating the mechanical properties, the handling characteristics, and the refilling ability of PMMA samples with both modified and unmodified CD microparticles. Future work for this study may be focused in two areas: 1) conducting more experiments evaluating more different aspects of the PMMA with both modified and unmodified CD microparticles, and 2) improving the mechanical and handling properties of PMMA cement mixed with CD microparticles.

\subsection{Investigating Other Properties of PMMA with CD Microparticles}

In order to ensure that the PMMA cement containing CD microparticles meets the ASTM F451-16 standards for clinically-used acrylic bone cement, there are a few more tests that can be performed (28). For example, rheometry, tensile test, and quantification of residual monomer after polymerization can be performed. Rheometry is generally conducted to investigate the handling and setting properties of PMMA cement by determining the viscosity of the curing PMMA dough (34). Since PMMA cement is also used in loadbearing applications, it can be subjected to a variety of stresses, so other mechanical tests such as the tensile test may also be performed to quantify the cements' mechanical properties under tension (35). Additionally, another interesting property to investigate is the surface roughness or the bonding between the PMMA cement and bone after the addition of $\mathrm{CD}$ microparticles. Below, two properties of particular interest, 
quantification of residual monomer and interfacial bond strength, are described in more detail.

The residual monomer content after polymerization is an important aspect to consider for different PMMA formulations. Studies have pointed out the presence of residual monomer after the polymerization or curing process of PMMA cement. Residual monomer has been shown to weaken the cement by acting as a plasticizer and hinders bone remodeling as monomer leaks out from the mantle into nearby bone tissue (36). Monomer toxicity also can lead to development of allergic reactions against the PMMA cement (5).

Based on the protocol from ASTM F451, the residual monomer content can be measured through a gas chromatography system. PMMA samples are soaked in vials of water, and aliquots are taken to evaluate the concentration of residual monomer (28).

Another area of interest is the effect of CD microparticles have on the surface roughness of the PMMA cement. Cements with rougher surface have been shown to bond stronger to the implant and to the native bone tissue $(37,38)$. Research groups have strived for a rougher PMMA cement surface in order to facilitate better bone healing and to improve implant stability by mixing in additives into the system $(38,39)$. Studies have shown that by increasing the surface roughness of the cement, cells such as osteoblasts may adhere better onto the cement allowing for osseointegration between the cement and native tissue (39). In addition to the osseointegration, microinterlocking between the irregular surface of cement and the native bone tissue have also been observed to increase the interfacial bond strength (40). 
To test the mechanical bond strength between PMMA cement and bone or implant, shear or pull-out tests and tensile tests can be performed $(37,40)$. Alternatively, a push-out system developed by our lab can also be used to test the bond strength between PMMA and bone tissue. An image of the push-out system can be found in Figure 10. In addition to test the mechanical bond strength between the PMMA and bone, an adhesion assay can also be conducted with osteoblasts to investigate the cytocompatibility of the PMMA formulation (39).

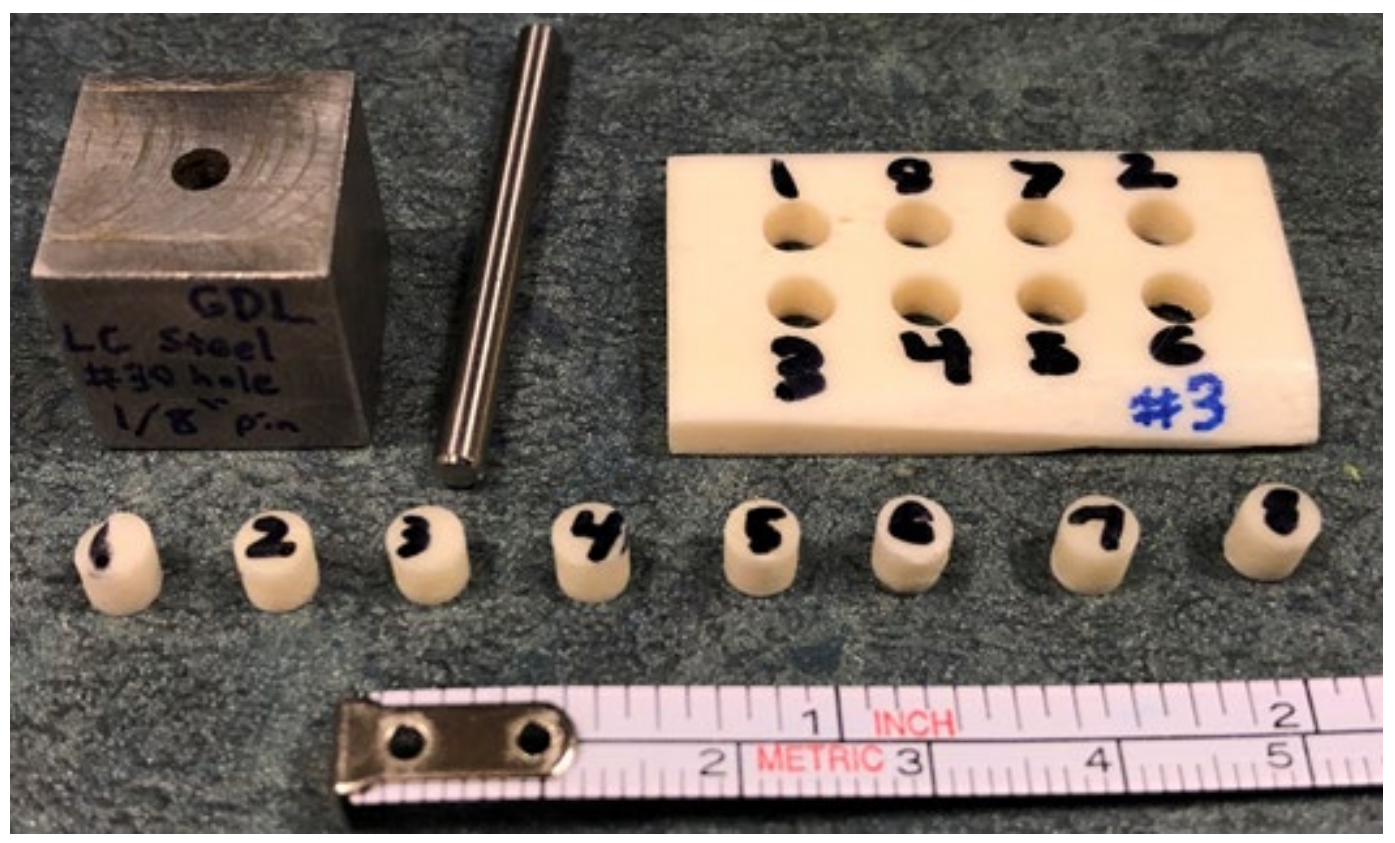

Figure 10. Image of push-out test system developed by our lab. The metallic components are for pushing out and collecting the cylindrical PMMA samples from the bone wafer. Cylindrical PMMA samples numbered 1 through 8 were pushed out from the corresponding holes in the bone wafer. 


\subsection{Improving Mechanical and Handling Properties of PMMA Containing CD Microparticles}

In addition to evaluating other properties of the PMMA formulation, potential future work can also focus on improving the current formulation, specifically, in the mechanical and handling properties of PMMA.

Even though the mechanical results presented in this study demonstrated that the substitution of unmodified $\beta-\mathrm{CD}$ microparticles for PMMA-CD was able to decrease the mechanical loss observed with addition of CD microparticles into PMMA cement, the formulations tested were not able to reach the ASTM standard minimum ultimate compressive strength of $70 \mathrm{MPa}$ (28). Additionally, the benefits of adding PMMA-CD instead of unmodified $\beta$-CD microparticles into PMMA cement become insignificant and appear to reach a threshold or plateau as more weight percentages of microparticles are mixed into the formulation. Specifically, the difference in ultimate compressive strengths of PMMA samples became less apparent when comparing PMMA samples with unmodified $\beta$-CD microparticles and samples with $26.3 \mathrm{kDa}$ PMMA-CD at $20 \mathrm{wt} \% \mathrm{CD}$ microparticles than at 10 or $15 \mathrm{wt} \% \mathrm{CD}$ microparticles (see Appendix).

To increase the mechanical strength of PMMA samples containing CD microparticles, filling CD microparticles prior to addition of liquid monomer may be a viable option. Prior studies have shown the possibility of liquid MMA monomer interacting with CD microparticles which may be the cause of loss in mechanical properties $(18,20)$. Scanning electron microscopic (SEM) images of PMMA samples with and without CD microparticles also showed less liquid coverage in PMMA samples (i.e. less presence of sharp spherical edges in the SEM images) with CD microparticles despite all samples 
having the same solid to liquid ratio (see Appendix). One prior study observed a 12\% increase in ultimate compressive strength when adding RMP-filled CD microparticles instead of adding empty CD microparticles (18). This difference may be due to a decrease in the interaction between the $\mathrm{CD}$ microparticles and the liquid monomer. By substituting empty modified CD microparticles used in this study with filled ones, the mechanical properties of PMMA cement may be further restored. Additionally, other properties (i.e. handling properties) may be studied with filled modified CD microparticles mixed in PMMA.

Another method to enhance the mechanical properties of PMMA samples with CD microparticles is to further modify $\mathrm{CD}$ microparticles to allow chemical interaction between the microparticles and PMMA. The modified CD microparticles used in this study were synthesized to only allow chain entanglement between the microparticles and PMMA, but this formulation was not able to reach the ASTM standards for the compressive strengths of acrylic cements (28) which suggests a need for further modification on CD microparticles. Functionalized CD microparticles that can be crosslinked to PMMA may be required to create a PMMA formulation with CD microparticles that reach the desirable mechanical properties. In addition to investigating methods for improving the mechanical properties of PMMA samples, the handling characteristics of different PMMA formulations can be further studied. Based on the results from this study, the addition of CD microparticles was able to decrease the high polymerization temperature and increase the short working time of PMMA. However, the relationship between the amount of CD microparticles and the handling characteristics of the PMMA formulation have not been investigated. Further 
studies in this area will allow others to determine the appropriate amount of CD microparticles to mix into the PMMA for the desired handling properties. 


\section{APPENDIX}

\section{Methods}

Scanning Electron Microscopy (SEM) Imaging

Images were taken to evaluate the microstructure for plain PMMA samples without microparticles, PMMA samples with $15 \mathrm{wt} \%$ unmodified $\beta$-CD microparticles, and PMMA samples with 15 wt\% 26.3 kDa PMMA-CD using a Scanning Electron Microscopy (SEM) (JSM-6510 Series, JEOL). These samples were prepared using a constant 2:1 ratio of solid to liquid monomer ratio $(\mathrm{g} / \mathrm{mL})$. Their fracture surfaces were sputter-coated with $20 \mathrm{~nm}$ of gold prior to imaging.

\section{Results}

SEM Imaging

SEM images were taken for plain PMMA samples without microparticles, PMMA samples with $15 \mathrm{wt} \%$ unmodified $\beta$-CD microparticles, and PMMA samples with $15 \mathrm{wt} \%$ 26.3 kDa PMMA-CD microparticles. These are shown in Figure S1.

Based on the SEM image for plain PMMA, it can be observed that the diameter of prepolymerized PMMA powder is around $55 \pm 9 \mu \mathrm{m}$ which is close to the $50 \mu \mathrm{m}$ diameter reported by Kuhn (41). The prepolymerized beads are also mostly covered by the polymerized liquid monomer except those that were closer to the edges of the PMMA sample. The liquid monomer coverage was determined by the lack of sharp spherical edges in the SEM images. No major differences were observed between the SEM images of samples with $15 \mathrm{wt} \% \beta$-CD microparticles and of those with $15 \mathrm{wt} \% 26.3 \mathrm{kDa}$ PMMA-CD microparticles. In comparison to samples without microparticles, PMMA samples with CD microparticles had less visible liquid monomer coverage (i.e. presence 
of more sharp spherical edges throughout the image) and showed presence of CD microparticles that have diameters of around $71 \pm 7 \mu \mathrm{m}$.

Plain PMMA

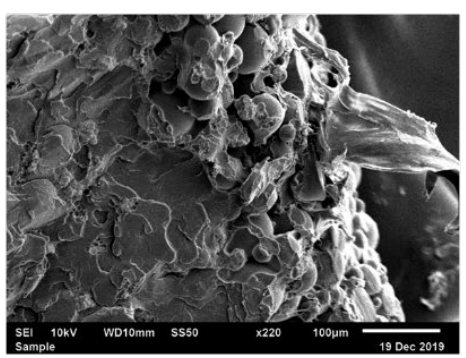

$15 \mathrm{wt} \% \beta-C D$

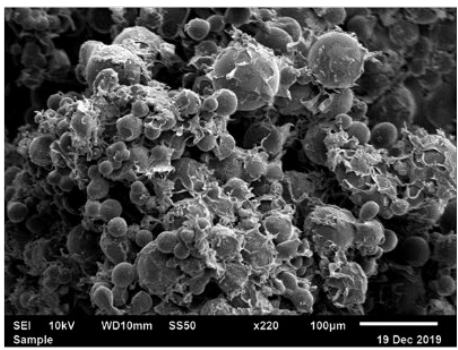

15 wt \% 26.3 kDa PMMA-CD

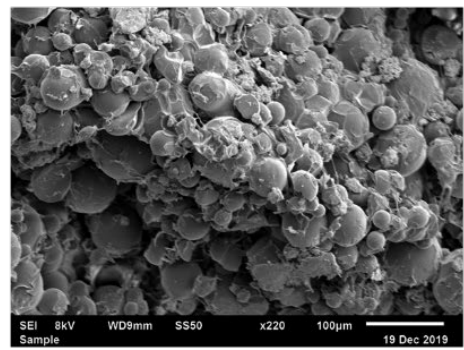

Figure S1. Scanning Electron Microscopy (SEM) images of plain PMMA without microparticles, PMMA with $15 \mathrm{wt} \% \beta-\mathrm{CD}$, and PMMA with $15 \mathrm{wt} \% 26.3 \mathrm{kDa}$ PMMA-CD. Scale bars $=100 \mu \mathrm{m}$.

\section{Compression Testing}

Compression tests were conducted on cylindrical PMMA samples to determine the mechanical properties of PMMA formulations with different types of CD microparticles. More specifically, relationship between the PMMA chain weights on modified CD microparticles and mechanical properties were investigated by testing PMMA samples with $10 \mathrm{wt} \%$ of microparticles (unmodified $\beta-\mathrm{CD}, 3.8 \mathrm{kDa}$ PMMA $\beta$-CD, $12.2 \mathrm{kDa}$ PMMA $\beta$-CD, $26.3 \mathrm{kDa}$ PMMA $\beta$-CD). The numerical results are presented in Table S1. Table S1 also includes mechanical properties of plain PMMA sample without microparticles as a negative control.

Table S1. Mechanical properties of PMMA samples without microparticles and PMMA samples with $10 \mathrm{wt} \% \beta$-CD microparticles (unmodified $\beta$-CD, 3.8kDa PMMA-CD, $12.2 \mathrm{kDa}$ PMMA-CD, or $26.3 \mathrm{kDa}$ PMMA-CD) based on compression testing $(\mathrm{n} \geq 3)$. Statistically significant difference 
of samples relative to PMMA with $10 \mathrm{wt} \%$ empty $\beta$-CD $(\dagger)(\mathrm{p}<0.05)$ and plain PMMA without microparticles $(\ddagger)(\mathrm{p}<0.05)$.

\begin{tabular}{|c|c|c|c|c|c|}
\hline $\begin{array}{l}\text { Compression } \\
\text { Test Specimen }\end{array}$ & $\begin{array}{l}\text { Ultimate } \\
\text { Compressive } \\
\text { Strength } \\
\text { (MPa) } \\
\end{array}$ & $\begin{array}{l}\text { Modulus } \\
\text { (MPa) }\end{array}$ & $\begin{array}{l}\text { Work to } \\
\text { Peak } \\
\text { Load (J) }\end{array}$ & $\begin{array}{l}\text { Strain to } \\
\text { Peak } \\
\text { Load }\end{array}$ & $\begin{array}{l}\text { Absorbed } \\
\text { Energy per } \\
\text { unit volume } \\
\left(\mathrm{J} / \mathrm{cm}^{\wedge} 3\right) \\
\end{array}$ \\
\hline $\begin{array}{l}10 w t \% 3.8 k D a \\
P M M A \beta-C D\end{array}$ & $58.89 \pm 2.90 \ddagger$ & $\begin{array}{l}1876.74 \pm \\
34.98+t\end{array}$ & $\begin{array}{l}0.870 \pm \\
0.056 \dagger\end{array}$ & $\begin{array}{l}0.056 \pm \\
0.001 \uparrow t\end{array}$ & $2.07 \pm 0.10 t t$ \\
\hline $\begin{array}{l}10 w t \% \\
12.2 \mathrm{kDa} \\
P M M A \beta-C D\end{array}$ & $60.89 \pm 2.79+t$ & $\begin{array}{l}1893.69 \pm \\
62.55+t\end{array}$ & $\begin{array}{l}0.815 \pm \\
0.008+t\end{array}$ & $\begin{array}{l}0.054 \pm \\
0.001 \dagger t\end{array}$ & $2.09 \pm 0.03 \dagger t$ \\
\hline $\begin{array}{l}10 w t \% \\
26.3 k D a \\
P M M A \beta-C D\end{array}$ & $62.09 \pm 1.48+t$ & $\begin{array}{l}1973.45 \pm \\
55.61 \dagger t\end{array}$ & $\begin{array}{l}0.864 \pm \\
0.026 \dagger\end{array}$ & $\begin{array}{l}0.0524 \pm \\
0.0002 \ddagger\end{array}$ & $2.09 \pm 0.04 \dagger \dagger$ \\
\hline $\begin{array}{l}10 w t \% \text { empty } \\
\beta-C D\end{array}$ & $54.65 \pm 2.56$ & $\begin{array}{l}1784.47 \pm \\
42.30 \ddagger\end{array}$ & $\begin{array}{l}0.721 \pm \\
0.040 \ddagger\end{array}$ & $\begin{array}{l}0.050 \pm \\
0.001 \ddagger\end{array}$ & $1.87 \pm 0.10 \ddagger$ \\
\hline Plain PMMA & $72.40 \pm 2.17 \dagger$ & $\begin{array}{l}2254.45 \pm \\
69.27 \dagger\end{array}$ & $\begin{array}{l}0.890 \pm \\
0.044 \dagger\end{array}$ & $\begin{array}{l}0.051 \pm \\
0.001 \dagger\end{array}$ & $2.26 \pm 0.11 \dagger$ \\
\hline
\end{tabular}

As modified CD microparticles with heavier PMMA chain weights were added into the PMMA cement, the ultimate compressive strengths and the modulus of the PMMA formulation tended to become more similar to those of plain PMMA without CD microparticles. Interestingly, the work to peak load and absorbed energy per unit volume significantly increased with the substitution of unmodified $\beta$-CD to modified CD (i.e. 3.8 kDa PMMA-CD, 12.2 kDa PMMA-CD, and 26.3 kDa PMMA-CD) $(\mathrm{p}<0.05)$ but did not have an apparent correlation with the PMMA chain weights attached onto the modified CD microparticles.

In addition to investigating the mechanical properties of samples with different types of CD microparticles, the relationship between the amount of microparticles added and the mechanical properties of PMMA samples were also evaluated. This relationship was tested with both PMMA samples that contained unmodified $\beta$-CD microparticles and 
PMMA samples that contained modified $\beta$-CD microparticles (i.e. $26.3 \mathrm{kDa}$ PMMACD). Plain PMMA samples that do not contain microparticles are also tested as negative control. The results are shown in Table S2.

Table S2. Mechanical properties of PMMA samples without and with 5 to $20 \mathrm{wt} \% \mathrm{CD}$ microparticles (unmodified $\beta$-CD or $26.3 \mathrm{kDa}$ PMMA-CD). Aside from plain PMMA without microparticles, the $2 \%$ offset method was used to determine the mechanical properties of samples $(\mathrm{n} \geq 3)$. Statistical significance is relative to PMMA samples with same $\mathrm{wt} \%$ of unmodified $\beta$-CD $(\dagger)(\mathrm{p}<0.05)$.

\begin{tabular}{|c|c|c|c|c|c|}
\hline $\begin{array}{l}\text { Compression } \\
\text { Test Specimen }\end{array}$ & $\begin{array}{l}\text { Ultimate } \\
\text { Compressive } \\
\text { Strength } \\
\text { (MPa) }\end{array}$ & $\begin{array}{l}\text { Modulus } \\
\text { (MPa) }\end{array}$ & $\begin{array}{l}\text { Work to } \\
\text { Peak } \\
\text { Load } \\
\text { (J) }\end{array}$ & $\begin{array}{l}\text { Strain } \\
\text { to Peak } \\
\text { Load }\end{array}$ & $\begin{array}{l}\text { Absorbed } \\
\text { Energy per } \\
\text { Unit Volume } \\
\left(\mathbf{J} / \mathrm{cm}^{\wedge} 3\right)\end{array}$ \\
\hline $5 w t \% \beta-C D$ & $61.52 \pm 6.76$ & $\begin{array}{l}1939.2 \pm \\
82.3\end{array}$ & $\begin{array}{l}0.825 \pm \\
0.132\end{array}$ & $\begin{array}{l}0.054 \pm \\
0.003\end{array}$ & $2.16 \pm 0.29$ \\
\hline $10 w t \% \beta-C D$ & $53.01 \pm 1.67$ & $\begin{array}{l}1651.3 \pm \\
191.4\end{array}$ & $\begin{array}{l}0.696 \pm \\
0.042\end{array}$ & $\begin{array}{l}0.054 \pm \\
0.005\end{array}$ & $1.82 \pm 0.11$ \\
\hline $15 w t \% \beta-C D$ & $47.25 \pm 2.05$ & $\begin{array}{l}1494.2 \pm \\
139.8\end{array}$ & $\begin{array}{l}0.653 \pm \\
0.067\end{array}$ & $\begin{array}{l}0.055 \pm \\
0.003\end{array}$ & $1.65 \pm 0.15$ \\
\hline $20 w t \% \beta-C D$ & $46.13 \pm 4.49$ & $\begin{array}{l}1480.4 \pm \\
133.3\end{array}$ & $\begin{array}{l}0.658 \pm \\
0.076\end{array}$ & $\begin{array}{l}0.054 \pm \\
0.002\end{array}$ & $1.59 \pm 0.20$ \\
\hline $\begin{array}{l}5 w t \% 26.3 \mathrm{kDa} \\
\text { PMMA-CD }\end{array}$ & $61.72 \pm 3.78$ & $\begin{array}{l}1924.8 \pm \\
146.8\end{array}$ & $\begin{array}{l}0.844 \pm \\
0.083\end{array}$ & $\begin{array}{l}0.055 \pm \\
0.002\end{array}$ & $2.16 \pm 0.17$ \\
\hline $\begin{array}{l}10 w t \% 26.3 \mathrm{kDa} \\
\text { PMMA-CD }\end{array}$ & $57.03 \pm 3.01 \dagger$ & $\begin{array}{l}1823.0 \pm \\
60.8\end{array}$ & $\begin{array}{l}0.795 \pm \\
0.063 \dagger\end{array}$ & $\begin{array}{l}0.053 \pm \\
0.001\end{array}$ & $1.97 \pm 0.15$ \\
\hline $\begin{array}{l}15 w t \% 26.3 \mathrm{kDa} \\
P M M A-C D\end{array}$ & $52.19 \pm 2.97 \dagger$ & $\begin{array}{l}1678.1 \pm \\
84.1 \dagger\end{array}$ & $\begin{array}{l}0.715 \pm \\
0.047\end{array}$ & $\begin{array}{l}0.053 \pm \\
0.001\end{array}$ & $1.78 \pm 0.11$ \\
\hline $\begin{array}{l}20 w t \% 26.3 k D a \\
\text { PMMA-CD }\end{array}$ & $44.84 \pm 1.66$ & $\begin{array}{l}1462.7 \pm \\
66.8\end{array}$ & $\begin{array}{l}0.629 \pm \\
0.032\end{array}$ & $\begin{array}{l}0.054 \pm \\
0.001\end{array}$ & $1.51 \pm 0.09$ \\
\hline
\end{tabular}




\begin{tabular}{llllll}
\hline Plain PMMA & $70.01 \pm 5.28$ & $1969.4 \pm$ & $0.943 \pm$ & $0.053 \pm$ & $2.31 \pm 0.26$ \\
& & 196.5 & 0.099 & 0.001 &
\end{tabular}

General negative trends were observed in all mechanical properties aside from strain to peak load (i.e. the ultimate compressive strength, modulus, work to peak load, and absorbed energy per unit volume) as more CD microparticles, both unmodified and modified, were added into the PMMA system. However, the decrease in these mechanical properties was more prominent in PMMA samples containing unmodified $\beta$ CD microparticles than in samples with modified CD (i.e. 26.3 kDa PMMA-CD) when between 10 to $15 \mathrm{wt} \%$ microparticles were added into the PMMA. It is important to note that PMMA samples with $20 \mathrm{wt} \%$ microparticles, both unmodified and modified CD, were fundamentally different from the other PMMA samples and tended to disintegrate more easily in comparison to others.

Further investigation has been made with similar formulation (i.e. $5 \mathrm{wt} \%, 10 \mathrm{wt} \%, 15$ $\mathrm{wt} \%$, and $20 \mathrm{wt} \%$ of microparticles in PMMA system) but with a constant solid to liquid monomer ratio in the system. It has been hypothesized that the decrease in ultimate compressive strengths observed was due to the increase in the solid to liquid ratio in the PMMA formulation $(20,26)$ which also may have contributed to the lack of difference in compressive strengths between PMMA that contained $20 \mathrm{wt} \%$ unmodified $\beta$-CD microparticles and PMMA that contained $20 \mathrm{wt} \% 26.3 \mathrm{kDa}$ PMMA-CD found in this study. To test this hypothesis, parts (between 5 to $20 \mathrm{wt} \%$ ) of the $2 \mathrm{~g}$ prepolymerized PMMA beads were replaced by unmodified $\beta$-CD microparticles or $26.3 \mathrm{kDa}$ PMMA$\mathrm{CD}$ before adding $1 \mathrm{~mL}$ of liquid monomer to form PMMA samples with constant 2:1 ratio $(\mathrm{g} / \mathrm{mL})$ of solid to liquid monomer. Compression tests were conducted in accordance to the other compression tests that had been done in this study (refer to 
Section 2.2.4). The results for the ultimate compressive strengths of PMMA samples with between 0 to $20 \mathrm{wt} \% \mathrm{CD}$ microparticles both unmodified and modified (i.e. $26.3 \mathrm{kDa}$ PMMA-CD) are shown below in Figure S2.

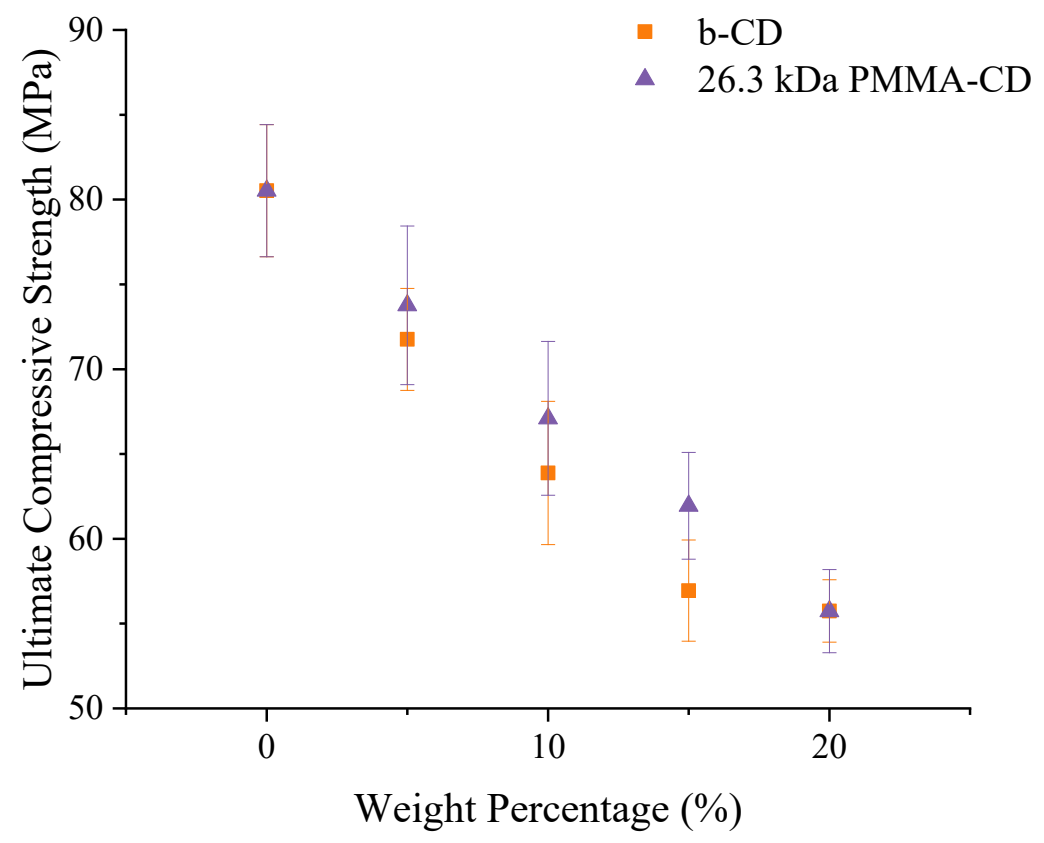

Figure S2. Ultimate compressive strengths of PMMA samples with between 0 to $20 \mathrm{wt} \% \mathrm{CD}$ microparticles (unmodified $\beta$-CD microparticles or $26.3 \mathrm{kDa}$ PMMA-CD) at constant liquid monomer to solid ratio.

Similar to the results from the compression tests reported earlier on this study for PMMA samples with between 0 to $20 \mathrm{wt} \% \mathrm{CD}$ microparticles at different solid to liquid ratios (refer to Section 3.1 or Figure 4), a general negative trend was found between the ultimate compressive strengths of the PMMA samples and the weight percentages of CD microparticles, regardless of modification, added into the PMMA. The difference in compressive strengths between the two formulations (i.e. PMMA with unmodified $\beta$-CD microparticles and PMMA with same weight percentage of microparticles but modified 
(26.3 kDa PMMA-CD)) tended to be smaller and insignificant $(\mathrm{p}>0.05)$ in comparison to the ones reported with PMMA samples that had increasing solid to liquid monomer ratio (refer to Section 3.1 or Figure 4). Interestingly, similar results were obtained at 20 wt $\%$ CD microparticles where no apparent difference in ultimate compressive strengths was found between PMMA samples that contained $\beta-\mathrm{CD}$ microparticles and those that contained 26.3 kDa PMMA-CD.

\section{Handling Characteristics}

Handling characteristics of different PMMA samples (i.e. plain PMMA without microparticles, PMMA with $10 \mathrm{wt} \%$ unmodified $\beta-\mathrm{CD}$ microparticles, and PMMA with $10 \mathrm{wt} \% 26.3 \mathrm{kDa}$ PMMA-CD microparticles) were evaluated by recording the temperature profile of each sample after the determination of their dough times. Temperature profiles of PMMA samples with $10 \mathrm{wt} \% 26.3 \mathrm{kDa}$ PMMA-CD microparticles $(n=3)$ and representative temperature profiles (i.e. profiles that had the closest maximum polymerization temperature, setting temperature, and setting time to the average values) of PMMA samples with no microparticles and PMMA samples with 10 wt $\%$ unmodified CD microparticles are presented in Figure S3. 


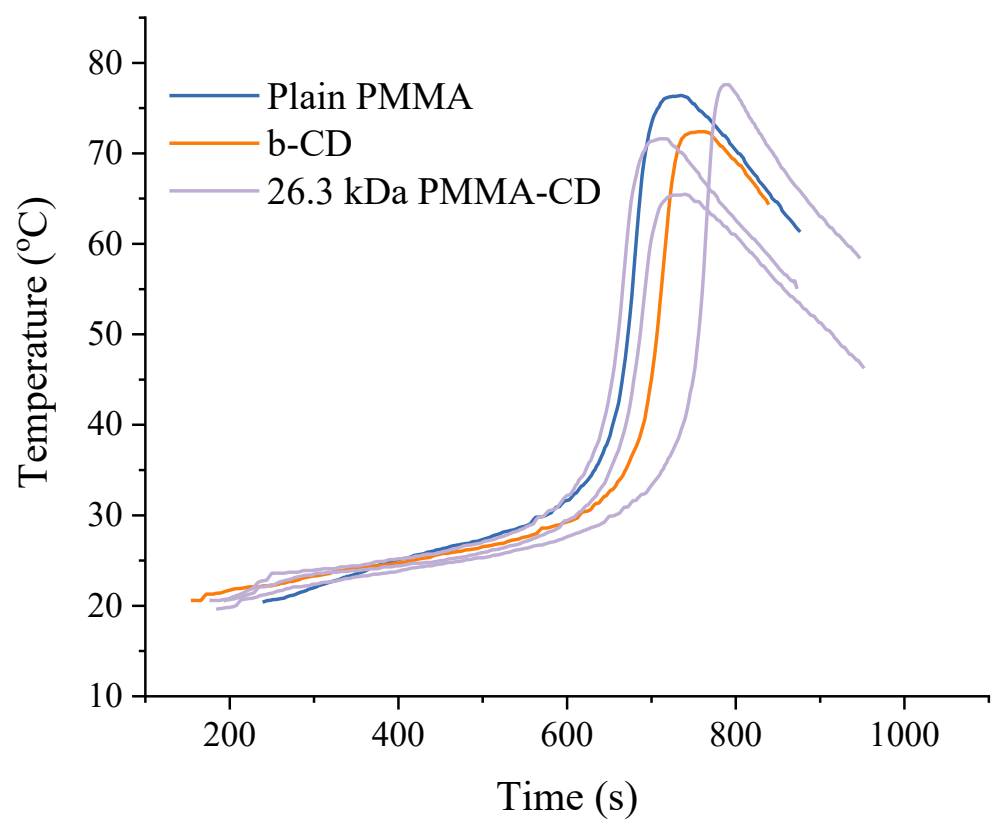

Figure S3. Representative temperature profiles of different PMMA formulations (plain PMMA without microparticles and PMMA with $10 \mathrm{wt} \%$ unmodified $\beta$-CD microparticles). Temperature profiles of PMMA with $10 \mathrm{wt} \% 26.3 \mathrm{kDa}$ PMMA-CD microparticles (n=3) are also shown.

Temperature profiles of all samples (i.e. plain PMMA without microparticles, PMMA with $10 \mathrm{wt} \%$ unmodified $\beta$-CD microparticles, and PMMA with $10 \mathrm{wt} \% 26.3 \mathrm{kDa}$ PMMA) generally followed a similar trend where the temperature initially increased at a slower rate followed by a sharp rise in temperature before reaching the peak maximum exothermic polymerization temperature. A delay in the rise of temperature and a lower peak temperature were observed with the addition of $10 \mathrm{wt} \%$ unmodified $\beta$-CD microparticles into plain PMMA without microparticles. More variance was observed in the temperature profiles of PMMA samples with $10 \mathrm{wt} \% 26.3 \mathrm{kDa}$ PMMA which may be due to the aggregation of microparticles. Increasing the sample size for PMMA samples 
with $10 \mathrm{wt} \% 26.3 \mathrm{kDa}$ PMMA may be required to achieve more consistent temperature profiles. 


\section{BIBLIOGRAPHY}

1. Springer BD, Cahue S, Etkin CD, Lewallen DG, Mcgrory BJ. Arthroplasty Today Infection burden in total hip and knee arthroplasties : an international registrybased perspective. Arthroplast Today. 2017;3(2):137-40.

2. Letchmanan K, Shen SC, Ng WK, Kingshuk P, Shi Z, Wang W, et al. Mechanical properties and antibiotic release characteristics of poly(methyl methacrylate)-based bone cement formulated with mesoporous silica nanoparticles. J Mech Behav Biomed Mater [Internet]. 2017;72(May):163-70. Available from: http://dx.doi.org/10.1016/j.jmbbm.2017.05.003

3. Seligson D, Berling S. Antibiotic-laden PMMA bead chains for the prevention of infection in compound fractures: current state of the art. Eur J Orthop Surg Traumatol. 2015;25(6):969-74.

4. Hendriks JGE, Horn JR Van, Mei HC Van Der, Busscher HJ. Backgrounds of antibiotic-loaded bone cement and prosthesis-related infection. Biomaterials. 2004;25(3):545-56.

5. Vaishya R, Chauhan M, Vaish A. Bone cement. J Clin Orthop Trauma [Internet]. 2013;4(4):157-63. Available from: http://dx.doi.org/10.1016/j.jcot.2013.11.005

6. Ayre WN, Birchall JC, Evans SL, Denyer SP. A novel liposomal drug delivery system for PMMA bone cements. J Biomed Mater Res Part B. 2016;104B:1510 24.

7. Carli A V., Sethuraman AS, Bhimani SJ, Ross FP, Bostrom MPG. Selected HeatSensitive Antibiotics Are Not Inactivated During Polymethylmethacrylate Curing and Can Be Used in Cement Spacers for Periprosthetic Joint Infection. J Arthroplasty [Internet]. 2018;33(6):1930-5. Available from: https://doi.org/10.1016/j.arth.2018.01.034

8. Jiranek WA, Hanssen AD, Greenwald AS. Antibiotic-Loaded Bone Cement for Infection Prophylaxis in Total Joint Replacement. J Bone Jt Surg. 2006;88A(11):2487-500.

9. Bistolfi A, Massazza G, Vern E, Mass A, Deledda D, Ferraris S, et al. AntibioticLoaded Cement in Orthopedic Surgery: A Review. ISRN Orthop. 2011;2011.

10. Oh EJ, Oh SH, Lee IS, Kwon OS, Lee JH. Antibiotic-eluting hydrophilized PMMA bone cement with prolonged bactericidal effect for the treatment of osteomyelitis. J Biomater Appl. 2015;30(10):1534-44.

11. Webb JCJ, Spencer RF. The role of polymethylmethacrylate bone cement in modern orthopaedic surgery. J Bone Jt Surg - Br Vol [Internet]. 2007;89$\mathrm{B}(7): 851-7$. Available from: http://www.bjj.boneandjoint.org.uk/cgi/doi/10.1302/0301-620X.89B7.19148 
12. Anagnostakos K, Meyer C. Antibiotic elution from hip and knee acrylic bone cement spacers: A systematic review. Biomed Res Int. 2017;2017(November 2016).

13. Shen SC, Ng WK, Dong YC, Ng J, Tan RBH. Nanostructured material formulated acrylic bone cements with enhanced drug release. Mater Sci Eng C [Internet]. 2016;58:233-41. Available from: http://dx.doi.org/10.1016/j.msec.2015.08.011

14. Al Thaher Y, Perni S, Prokopovich P. Nano-carrier based drug delivery systems for sustained antimicrobial agent release from orthopaedic cementous material. Adv Colloid Interface Sci [Internet]. 2017;249(April):234-47. Available from: https://doi.org/10.1016/j.cis.2017.04.017

15. Hanssen AD. Prophylactic use of antibiotic bone cement: An emerging standard In opposition. J Arthroplasty. 2004;19(4 SUPPL. 1):73-7.

16. Wei W, Abdullayev E, Hollister A, Mills D, Lvov YM. Clay nanotube/poly(methyl methacrylate) bone cement composites with sustained antibiotic release. Macromol Mater Eng. 2012;297(7):645-53.

17. Wang NX, von Recum HA. Affinity-Based Drug Delivery. Macromol Biosci. 2011;11:321-32.

18. Cyphert EL, Learn GD, Hurley SK, Lu C, von Recum HA. An Additive to PMMA Bone Cement Enables Postimplantation Drug Refilling, Broadens Range of Compatible Antibiotics, and Prolongs Antimicrobial Therapy. Adv Healthc Mater [Internet]. 2018 Nov;7(21):1800812. Available from:

http://doi.wiley.com/10.1002/adhm.201800812

19. Pahlevanzadeh F, Bakhsheshi-Rad HR, Hamzah E. In-vitro biocompatibility, bioactivity, and mechanical strength of PMMA-PCL polymer containing fluorapatite and graphene oxide bone cements. J Mech Behav Biomed Mater [Internet]. 2018;82(December 2017):257-67. Available from:

https://doi.org/10.1016/j.jmbbm.2018.03.016

20. Cyphert EL, Lu C, Marques DW, Learn GD, von Recum HA. Combination Antibiotic Delivery in PMMA Provides Sustained Broad-Spectrum Antimicrobial Activity and Allows for Postimplantation Refilling. Biomacromolecules [Internet]. 2020 Feb 10;21(2):854-66. Available from:

https://pubs.acs.org/doi/10.1021/acs.biomac.9b01523

21. Santos JGF, Pita VJRR, Melo PA, Nele M, Pinto JC. Production of bone cement composites: Effect of fillers, co-monomer and particles properties. Brazilian J Chem Eng. 2011;28(2):229-41.

22. Sa Y, Yang F, Wang Y, Wolke JGC, Jansen JA. Modifications of Poly(Methyl Methacrylate) Cement for Application in Orthopedic Surgery. In: Chun H, Park C, Kwon I, Khang G, editors. Cutting-Edge Enabling Technologies for Regenerative 
Medicine [Internet]. Springer, Singapore; 2018. p. 119-34. Available from: http://link.springer.com/10.1007/978-981-13-0950-2_7

23. Tai CL, Lai PL, Lin W De, Tsai TT, Lee YC, Liu MY, et al. Modification of Mechanical Properties, Polymerization Temperature, and Handling Time of Polymethylmethacrylate Cement for Enhancing Applicability in Vertebroplasty. Biomed Res Int. 2016;2016.

24. Koh BTH, Tan JH, Ramruttun AK, Wang W. Effect of storage temperature and equilibration time on polymethyl methacrylate (PMMA) bone cement polymerization in joint replacement surgery. J Orthop Surg Res [Internet]. 2015;10(1):1-6. Available from: http://dx.doi.org/10.1186/s13018-015-0320-7

25. Perry KI, Hanssen AD. Orthopaedic Infection. J Am Acad Orthop Surg [Internet]. 2017 Feb;25(suppl 1):S4-6. Available from:

http://content.wkhealth.com/linkback/openurl?sid=WKPTLP:landingpage\&an=00 124635-201702001-00002

26. Cyphert EL, Learn GD, Hurley SK, Lu C, von Recum HA. An Additive to PMMA Bone Cement Enables Postimplantation Drug Refilling, Broadens Range of Compatible Antibiotics, and Prolongs Antimicrobial Therapy. Adv Healthc Mater [Internet]. 2018 Nov;7(21):1800812. Available from: http://doi.wiley.com/10.1002/adhm.201800812

27. Grafmiller KT, Zuckerman ST, Petro C, Liu L, von Recum HA, Rosen MJ, et al. Antibiotic-releasing microspheres prevent mesh infection in vivo. J Surg Res [Internet]. 2016;206(1):41-7. Available from: http://dx.doi.org/10.1016/j.jss.2016.06.099

28. ASTM International. Standard Specification for Acrylic Bone Cement F451-16. 2016;

29. Ranjan RK, Kumar M, Kumar R, Ali MF. Bone cement. Int J Orthop Sci [Internet]. 2017 Oct 1;3(4b):79-82. Available from: http://www.orthopaper.com/archives/?year=2017\&vol=3\&issue=4\&part=B\&Artic leId $=562$

30. Cyphert EL, Zuckerman ST, Korley JN, von Recum HA. Affinity interactions drive post-implantation drug filling, even in the presence of bacterial biofilm. Acta Biomater [Internet]. 2017 Jul;57:95-102. Available from: https://linkinghub.elsevier.com/retrieve/pii/S1742706117302428

31. Chavali R, Resijek R, Knight SK, Choi IS. Extending polymerization time of polymethylmethacrylate cement in percutaneous vertebroplasty with ice bath cooling. Am J Neuroradiol. 2003;24(3):545-6.

32. Samad HA, Jaafar M. Effect of polymethyl methacrylate (PMMA) powder to liquid monomer $(\mathrm{P} / \mathrm{L})$ ratio and powder molecular weight on the properties of 
PMMA cement. Polym - Plast Technol Eng. 2009;48(5):554-60.

33. Pascual B, Gurruchaga M, Ginebra MP, Gil FJ, Planell JA, Gonĩ I. Influence of the modification of $\mathrm{P} / \mathrm{L}$ ratio on a new formulation of acrylic bone cement. Biomaterials. 1999;20(5):465-74.

34. Farrar DF, Rose J. Rheological properties of PMMA bone cements during curing. Biomaterials [Internet]. 2001 Nov;22(22):3005-13. Available from: https://linkinghub.elsevier.com/retrieve/pii/S0142961201000473

35. Lee C. Properties of Bone Cement: The Mechanical Properties of PMMA Bone Cement. In: The Well-Cemented Total Hip Arthroplasty. 2005. p. 60-6.

36. Vallo CI. Residual monomer content in bone cements based on poly(methyl methacrylate). Polym Int [Internet]. 2000 Aug;49(8):831-8. Available from: http://doi.wiley.com/10.1002/10970126\%28200008\%2949\%3A8\%3C831\%3A\%3AAID-PI462\%3E3.0.CO\%3B2-3

37. Walsh WR, Svehla MJ, Russell J, Saito M, Nakashima T, Gillies RM, et al. Cemented fixation with PMMA or Bis-GMA resin hydroxyapatite cement: Effect of implant surface roughness. Biomaterials. 2004;25(20):4929-34.

38. Khandaker M, Li Y. Interfacial Fracture Strength Measurement of Bone-PMMA Cement Constructs. In: Proceedings of the International Conference on Mechanical Engineering. Dhaka, Bangladesh: ICME2011; 2011.

39. Ricker A, Liu-Snyder P, Webster TJ. The influence of nano $\mathrm{MgO}$ and $\mathrm{BaSO} 4$ particle size additives on properties of PMMA bone cement. Int J Nanomedicine. 2008;3(1):125-32.

40. Skripitz R, Aspenberg P. Attachment of PMMA cement to bone: Force measurements in rats. Biomaterials. 1999;20(4):351-6.

41. Kuhn K. Bone Cements: Up-to-Date Comparison of Physical and Chemical Properties of Commercial Materials. Springer-Verlag Berlin and Heidelberg GmbH \& Co. KG; 2011. 272 p. 